\title{
Early history of extended irreversible thermodynamics (1953-1983): An exploration beyond local equilibrium and classical transport theory
}

\author{
G. Lebon ${ }^{1}$ and D. Jou ${ }^{2, a}$ \\ 1 Département d'Astrophysique, Géophysique et Océanographie, Bâtiment B5, Liège Uni- \\ versity, 4000 Liège, Belgium \\ 2 Departament de Física, Universitat Autònoma de Barcelona, 08193 Bellaterra, Catalonia, \\ Spain
}

Received 22 May 2014 / Received in final form 4 November 2014

Published online 23 December 2014

(C) EDP Sciences, Springer-Verlag 2014

\begin{abstract}
This paper gives a historical account of the early years (19531983) of extended irreversible thermodynamics (EIT). The salient features of this formalism are to upgrade the thermodynamic fluxes of mass, momentum, energy, and others, to the status of independent variables, and to explore the consistency between generalized transport equations and a generalized version of the second law of thermodynamics. This requires going beyond classical irreversible thermodynamics by redefining entropy and entropy flux. EIT provides deeper foundations, closer relations with microscopic formalisms, a wider spectrum of applications, and a more exciting conceptual appeal to non-equilibrium thermodynamics. We first recall the historical contributions by Maxwell, Cattaneo, and Grad on generalized transport equations. A thermodynamic theory wide enough to cope with such transport equations was independently proposed between 1953 and 1983 by several authors, each emphasizing different kinds of problems. In 1983, the first international meeting on this theory took place in Bellaterra (Barcelona). It provided the opportunity for the various authors to meet together for the first time and to discuss the common points and the specific differences of their previous formulations. From then on, a large amount of applications and theoretical confirmations have emerged. From the historical point of view, the emergence of EIT has been an opportunity to revisit the foundations and to open new avenues in thermodynamics, one of the most classical and well consolidated physical theories.
\end{abstract}

a e-mail: david.jou@uab.cat 


\section{Introduction}

The aim of this article is to present and to illustrate the early history and developments of a unified non-equilibrium thermodynamic approach that goes beyond local equilibrium and the classical transport laws. This formalism is presently known as "Extended Irreversible Thermodynamics"; the term "extended thermodynamics" is also used in the literature to denote thermodynamic theories incorporating the thermodynamic fluxes as independent variables. These theories include extended irreversible thermodynamics, rational extended thermodynamics, internal variables theory, and some approaches to kinetic theory. All of them will be discussed in this paper, albeit not in full extent, which would be impracticable, but focusing on their respective contributions to the development of the formalism. Extended thermodynamics has been the subject of several books and hundreds of articles and embraces a wide variety of phenomena, from heat, matter and electric transport to rheology, relativity, and cosmology. Modern technology strives towards miniaturized devices, high frequency, and strongly non-linear processes, which emphasizes the need to open the way to new conceptual frameworks generalizing the classical approaches of equilibrium and nonequilibrium thermodynamics with the perspective not only to cope with these new technological challenges, but also to suggest new practical inputs.

Extended irreversible thermodynamics (EIT) establishes a deep coupling between generalized transport equations including relaxation and non-local terms, and a generalized entropy and entropy flux, leading to a consistency of the transport equations with the second law of thermodynamics. This is not possible in the framework of classical irreversible thermodynamics, and therefore one has to generalize the notions of entropy and of entropy flux, together with a reformulation of the second law. The basic idea behind EIT is to take the fluxes as independent variables, in addition to the classical thermodynamic variables, to consider the transport equations as evolution equations for the fluxes, and to explore the corresponding contributions of the fluxes to the entropy and entropy flux. This approach asks for a deep reexamination of the foundations, namely, the definition of entropy (which in classical thermodynamics is only defined for equilibrium states), the meaning of temperature, the formulation of the second law at the mesoscopic level, and their relation with microscopic and macroscopic descriptions. This view also opens new ways towards the description of multi-scale phenomena, involving different time and length scales.

The idea of taking the fluxes as independent variables which began in EIT with the purpose to deal with some specific and highly specialized topics has turned into a much wider philosophy, which can nowadays be transferred to many other different disciplines.

EIT illustrates the relevance of new ideas in thermodynamics. This is in contrast with most current approaches, which are mainly interested in the dynamical aspects of the phenomena, where generalized transport equations are either proposed or derived from microscopic bases, or molecular dynamical simulations, without paying attention to fundamental problems as those related to entropy and the second law.

From a practical point of view, a mesoscopic approach as EIT, intermediate between the microscopic and the macroscopic ones, is useful because it leads to more detailed approximate equations than the classical ones are. It allows for much faster analyses than those carried out on a microscopic basis. Once having identified the most promising physical configurations and conditions, one can still go back to their microscopic foundations.

In the next sections, we present a survey of the evolution of EIT since its beginnings with Machlup and Onsager [Machlup 1953] and its first formulations up to 1983 [Casas-Vazquez 1984] when the formalism was seen as a consistent entity rather than a mere collection of unrelated parts. The generalization of the classical transport 
equations may be traced back to Maxwell who introduced constitutive equations with a relaxation term [Maxwell 1867]. He pointed out that the stress tensor in a viscous gas is decreasing at a rate depending on the nature of the system and the amount of the stress. However, Maxwell regarded this result as unimportant. The seminal work of Grad who sought to find non-normal solutions to the Boltzmann equation should also be underlined [Grad 1949]. Grad was the first to suggest that the fluxes of heat and diffusion are good candidates to be selected as independent state variables in the kinetic descriptions of dilute gases. His idea served to establish the microscopic foundations of EIT.

The present paper is organized as follows. In Section 2, we give a brief overview of the classical transport equations and place them in the framework of classical irreversible thermodynamics (CIT). We point out some of the deficiencies of CIT, in particular its failure to account for heat waves, relaxation effects, and viscoelasticity. Attempts to include relaxation effects in transport equations can be found in articles by Maxwell [Maxwell 1867], Cattaneo [Cattaneo 1948], Grad [Grad 1949], and Vernotte [Vernotte 1958] whose contributions are summarized in Section 3. In Section 4 we present the essential ideas underlying EIT for the readers unacquainted with them. The first attempt to place the relaxation effects into the framework of a macroscopic thermodynamic description was done by Machlup and Onsager [1953]. During the period 1953-1983, the foundations of EIT were more explicitly established and illustrated by a panoply of applications thanks to generally independent contributions by Nettleton, Müller, Gyarmati, Lambermont and Lebon, Lebon, Jou et al. [Nettleton 1959, 1960; Müller 1966, 1967; Gyarmati 1970; Lambermont 1973; Lebon 1978; Jou 1979, 1983], which are described in Section 5. Relativistic versions by Müller, Kranys, Israel and Pavon [Müller 1969; Kranys 1972; Israel, 1976; Pavon 1980] are discussed in Section 6. Kinetic theory and statistical mechanics foundations of EIT [Eu 1980; Garcia-Colin 1982; Luzzi 2001] are summarized in Section 7 and a short account of other non-equilibrium thermodynamic theories can be found in Section 8. A first international meeting bringing together the most prominent contributors to EIT and whose content is summarized in Section 9, took place at the Autonomous University of Barcelona in 1983. At this occasion, the several and various aspects were put on a common ground, and more ambitious and overarching versions of EIT began to be formulated. This was followed by the extension of the formalism to cover new applications and the involvement of a wider number of researchers. In that respect the period 1983-2014 represents a fruitful and exalting period with the publications of several books and dozens of articles. But we prefer to report on these advancements in future work, as the topics covered in the present paper are already rather complex.

While submitting the present work for publication, our attention was drawn to a review paper on the history of irreversible thermodynamics by Müller and Weiss [Müller 2012]. These authors present the history of irreversible thermodynamics from the vantage point of view of Rational Thermodynamics as promoted mainly by Coleman and Noll [Coleman 1963, 1964] and Truesdell [Truesdell 1969]. Less attention is given to the collective efforts of the several contributors to the development of the EIT formalism. In that respect, we suggest that the present review may be seen as a useful complement to that of Müller and Weiss.

\section{Classical irreversible thermodynamics and classical transport equations}

One of the exciting discoveries of physics in the 19th century was the formulation of mathematical laws describing the transport of heat, mass, electric charge, and 
momentum in continuous media. These laws went beyond reversible Newtonian mechanics and led to the description of irreversible phenomena, providing a different perspective in physics, closer to the observations in realistic set-ups. Though Newton himself had opened this way with his law on the cooling of bodies [Newton 1701] and his theory on viscous forces published in Principia (3rd edition 1726), the mathematical breakthrough came with Fourier's law for heat flow [Fourier 1822] according to which the heat flux is proportional to the temperature gradient. Note that Fourier's theory precedes Carnot's essay Sur la puissance motrice du feu [Carnot 1824], which is considered to be the stepping stone of thermodynamics. Both Fourier and Carnot worked in the problematic framework of the caloric theory, which was the accepted framework until 1850.

In the mid-19th century, Fourier's ideas were extended to other contexts. Fick generalized them for diffusion flow [Fick 1855] while the growing interest in electricity led Ohm to propose that the electric current is proportional to the gradient of the electrical potential [Ohm 1827]. In fluid mechanics, Stokes formulated the law presently known as Navier-Stokes relating the stress tensor and the velocity gradient tensor equation [Stokes 1851]. Furthermore, the interest in possible connections between heat and electric flows, which became manifest, for instance, in Joule's experiments [Joule 1841] on the heating effects of electrical currents, opened the way to a thorough exploration of couplings between these two flows, which culminated in the discovery of the thermoelectric effects [Seebeck 1821; Peltier 1839; Thomson 1854], which were shown to be closely related to each other. Other examples of couplings are provided by the thermo-osmotic effect, observed between heat and mass flow across membranes, or in liquid and gas mixtures with the Soret effect where a temperature gradient induces a matter motion [Soret 1879], or the Dufour effect where a mass concentration gradient generates a heat flux [Dufour 1873].

One of the aims of classical thermodynamics was to combine the concept of equilibrium states with transport theory, without paying much attention to timeevolution processes. Classical thermodynamics may be considered a consolidated theory from 1850 on, with the definition of absolute temperature by Thomson, later Lord Kelvin [Thomson 1848], and the formulations of the second law by Thomson and Clausius [Thomson 1851; Clausius 1854] who later on introduced the concept of entropy [Clausius 1865]. At the same period, significant contributions were made by Boltzmann who derived the celebrated transport equation [Boltzmann 1872] bearing his name. Boltzmann's main contribution is the statistical definition of entropy [Boltzmann 1895] (later) written as $S=k_{B} \ln W$ wherein $W$ is the number of possible microscopic arrangements among the distribution of $N$ atoms and $k_{B}$ Boltzmann's constant. Boltzmann's interpretation of entropy growth and irreversibility was the source of acrid conflicts. These fundamental contributions, together with Gibbs' masterwork [Gibbs 1875] "On the Equilibrium of Heterogeneous Substances", gave to thermodynamics a fully-fledged theoretical and practical body which since then has been one of the cornerstones of classical physics.

The foremost aim of the early authors of non-equilibrium thermodynamics was to construct a thermodynamic foundation to the phenomenological laws established in the 19th century by Fourier, Fick and Stokes. Pioneering works in this field are due to the two pioneers, Onsager and Prigogine, honored for their work with Nobel Prizes, in 1968 and 1977, respectively, further two papers by Eckart [Eckart 1940] and books by Denbigh, de Groot, Meixner and Reik, Haase and de Groot and Mazur [Denbigh 1950; de Groot 1951; Meixner 1959; Haase 1969; de Groot 1962]. These works constitute the gist of what is nowadays dubbed as "Classical Irreversible Thermodynamics" (CIT).

The basic idea of CIT is the local equilibrium hypothesis. According to it, the local and instantaneous relations between thermodynamic quantities in a system away from equilibrium are the same as for a uniform system in equilibrium. A quantitative 
evaluation of this approximation can be achieved through the Deborah number $D e=\tau_{m} / \tau_{M}$ with $\tau_{m}$, denoting the microscopic equilibration time, e.g. the time interval between two successive collisions among the particles of the system, which is of the order of $10^{-12} \mathrm{~s}$ in gases at atmospheric pressure or in solids, and the macroscopic characteristic time $\tau_{M}$ whose order of magnitude is related to the duration of an experiment, say about one second. For $D e \ll 1$, the local-equilibrium hypothesis is fully justified because the relevant variables evolve on a large time scale $\tau_{M}$ and do practically not change over the time $\tau_{m}$, but the hypothesis is not appropriate to describe situations characterized by $D e \gg 1$. Large values of $D e$ are found in systems with long relaxation times, like polymers, rarefied gases, superfluids and superconductors or in high-frequency or very fast phenomena, such as ultrasound propagation, shock waves, laser pulse heating of materials, nuclear collisions, etc. A consequence of the local equilibrium assumption is that all the variables defined in equilibrium, like entropy and temperature, remain unequivocally defined away from equilibrium, but they are allowed to vary with time and space. Another consequence is that the local state variables are related by the same state equations as in equilibrium. This means, in particular, that the Gibbs relation between entropy and the state variables remains valid locally for each value of the time $t$ and the position vector $\boldsymbol{r}$. For example, in the case of an $n$-component fluid of total mass $m$, the local Gibbs equation reads

$$
d s=T^{-1} d u+p T^{-1} d v-T^{-1} \sum_{k=1}^{n} \mu_{k} d c_{k},
$$

where $s$ is the specific entropy (per unit mass), $u$ the specific internal energy, $T$ the absolute temperature, $p$ the hydrostatic pressure, $v$ the specific volume which is related to the mass density $\rho$ by $v=1 / \rho, c_{k}=m_{k} / m$ the mass fraction of substance $k$, with $\mu_{k}$ its chemical potential. From the kinetic theory point of view, the local equilibrium hypothesis is justified only for conditions where the Maxwellian distribution is approximately valid.

The second cornerstone of CIT is to assume that the time evolution of entropy is governed by a local balance equation of the form

$$
\rho d s / d t=-\nabla \cdot J^{s}+\sigma^{s}
$$

the quantities $\boldsymbol{J}^{s}$ and $\sigma^{s}$ stand for the entropy flux vector and the rate of entropy production per unit volume respectively. In virtue of the second law of thermodynamics one has

$$
\sigma^{s} \geqslant 0
$$

where the inequality sign refers to irreversible processes and the equality sign to reversible ones or to equilibrium states. Note that this statement is more restrictive than the formulation of the second law in equilibrium thermodynamics, which only requires that in isolated systems, the global entropy of the final equilibrium state is higher or equal to its value in the initial equilibrium state, which does not exclude situations with entropy decreasing for short times, on the condition that in the final state, the entropy is larger than in the initial one. In contrast, expression (2.3) states that entropy production must increase at any time and any point in space.

Generalizing the second law to non-equilibrium states is not a trivial matter as entropy is not defined in non-equilibrium states, moreover, classical thermodynamics compares only entropy in the final and initial equilibrium states but does not provide unequivocal information about the behavior of the entropy in intermediate states during the process.

Combining the Gibbs equation and the entropy balance, it is found, after use is made of the conservation laws of energy, total mass and mass fraction, that $\sigma^{s}$ consists 
Table 1. Thermodynamic fluxes and forces in a one-component viscous fluid.

\begin{tabular}{cc}
\hline Fluxes & Forces \\
\hline $\boldsymbol{q}$ & $\nabla T$ \\
$p^{v}$ & $\nabla \cdot \boldsymbol{v}$ \\
$\boldsymbol{P}^{0 \boldsymbol{v}}$ & $\boldsymbol{V}^{0}$ \\
\hline
\end{tabular}

of a sum of products of so-called thermodynamic fluxes $J_{\alpha}$ and thermodynamic forces $X_{\alpha}$

$$
\sigma^{s}=\sum_{\alpha} J_{\alpha} X_{\alpha}
$$

The thermodynamic forces $X_{\alpha}$ are generally related to the gradients of the intensive variables (the name forces does not refer to truly mechanical forces, but to general factors driving changes in the system, in an analogous way as one could refer to social forces or intellectual forces driving a change in society), whereas the fluxes $J_{\alpha}$ can be identified with the fluxes of energy, mass, mass fractions, or momentum. In the case of a one-component fluid, expressions for $J_{\alpha}$ and $X_{\alpha}$ are given in Table 1, where $\boldsymbol{q}$ is the heat flux vector, $\boldsymbol{P}^{0 v}$ the traceless symmetric, second-order pressure tensor defined as $\boldsymbol{P}^{0 v}=\boldsymbol{P}^{v}-\left(\frac{1}{3} \operatorname{tr} \boldsymbol{P}^{v}\right) \boldsymbol{I}$, (upper index ${ }^{0}$ denotes the deviatoric part of the tensor and $\boldsymbol{I}$ the identity tensor), $p^{v}$ the scalar viscous pressure $\left(=(1 / 3) \operatorname{tr} \boldsymbol{P}^{v}\right), \boldsymbol{v}$ the velocity vector (not to be confused with the scalar specific volume $v$ ) and $\boldsymbol{V}$ the symmetric part of the velocity gradient tensor.

Experience indicates that the thermodynamic fluxes and forces are not independent but that there are relations between them, see, e.g. the Seebeck, Peltier, Dufour and Soret effects, the coupling of several chemical reactions in chemical kinetics, or the relations between chemical reactions and transport in biological systems. Moreover, the simplest way to ensure that the rate of entropy production $\sigma^{s}$ is positive definite is to assume that the fluxes are linear functions of the forces so that

$$
J_{\alpha}=\sum_{\beta} L_{\alpha \phi} X_{\beta}
$$

here the $L_{\alpha \beta}$ are so-called phenomenological coefficients. The flux-force relations (2.5) are named phenomenological relations, constitutive equations, or transport equations. Substitution of the linear flux-force relations (2.5) into expression (2.4) of the rate of entropy production yields the quadratic form

$$
\sigma^{s}=\sum_{\alpha, \beta} L_{\alpha \beta} X_{\alpha} X_{\beta} \geqslant 0
$$

According to standard results in algebra, e.g. Courant and Hilbert [Courant 1962], the necessary and sufficient conditions for $\sigma^{s} \geqslant 0$ are that the determinant $L_{\alpha \beta}+L_{\beta \alpha}$ and all its principal minors are nonnegative, from which it follows in particular that $L_{\alpha \alpha} \geqslant 0$. Other restrictions concern the symmetry properties of the phenomenological coefficients. It was proved by Onsager that, as a consequence of microscopic time reversibility (i.e. invariance of equations of motion of particles at the microscopic level with respect to time reversal), and the hypothesis of regression of fluctuations in the mean, that the coefficients $L_{\alpha \beta}$ are either symmetric [Onsager 1931] or skew symmetric [Casimir 1945] depending on the relative time-reversal property of fluxes and forces:

$$
L_{\alpha \beta}= \pm L_{\beta \alpha} .
$$


Applying the general results (2.4)-(2.6) to a one-component fluid leads to the following bilinear expression of the rate of entropy production per unit volume

$$
\sigma^{s}=\boldsymbol{q} \cdot \nabla T^{-1}-T^{-1} p^{v} \nabla \cdot \boldsymbol{v}-T^{-1} \boldsymbol{P}^{0 v}: \boldsymbol{V} \geqslant 0
$$

which suggests the formulation of the following linear transport equations:

$$
\begin{aligned}
\boldsymbol{q} & =L_{q q} \nabla T^{-1} \equiv-\lambda \nabla T, & & \text { (Fourier's law) } \\
p^{v} & =-l_{v v} T^{-1} \nabla \cdot \boldsymbol{v} \equiv-\zeta \nabla \cdot \boldsymbol{v}, & & \text { (Stokes' law) } \\
\boldsymbol{P}^{0 v} & =-L_{v v} T^{-1} \boldsymbol{V}^{0} \equiv-2 \eta \boldsymbol{V}^{0} & & \text { (Newton's law) }
\end{aligned}
$$

One recognizes in equation (2.9) Fourier's law by identifying the phenomenological coefficient $L_{q q} / T^{2}$ with the heat conductivity $\lambda$; expression (2.10) is Stokes relation after $l_{v v} / T$ is identified with the bulk viscosity $\zeta$, and finally (2.11) is Newton's law of hydrodynamics when $L_{v v} / T$ is set equal to $2 \eta$, with $\eta$ the dynamic shear viscosity. After substitution of (2.9)-(2.11) in (2.8), it is concluded from $\sigma^{s}>0$ that $\lambda>0$, $\zeta>0, \eta>0$. The above results are important as they provide a thermodynamic background to the thermo-hydrodynamic transport relations. Other examples of fluxforce relations are Fick's law of diffusion and Ohm's law in electricity.

Besides providing a well-defined framework for the classical transport equations, CIT proves also to be useful as a basis for variational formulations. These have always played a central role in physics, recall the least action principle in rational mechanics or Fermat's principle in optics, but also in CIT Prigogine's minimum entropy production's principle. Prigogine's principle states that the total entropy production $P$ decreases over time and is minimal at a stationary state, but it only holds under rather restrictive conditions such as time-independent boundary conditions, linear flux-force relations, and constant and symmetric phenomenological coefficients. A generalization of Prigogine's principle in the framework of EIT was proposed by Lebon and Dauby to account for the non-local heat conduction equation [Lebon 1990] derived by Guyer and Krumhansl [Guyer 1966]. Lebon and Dauby's formulation served to establish the relevant heat slip boundary conditions frequently used in microfluidics and nanomaterials science [Jou 2010]. Going back to CIT, Prigogine's principle was extrapolated under the form of the so-called local potential [Glansdorff 1964, 1971] which opened the way to the study of the so-called dissipative structures, a term introduced by Prigogine. Dissipative structures take the form of well-ordered spatio-temporal patterns appearing far from equilibrium after the thermodynamic forces have reached a critical point. Typical examples are provided by Bénard's cells [Bénard 1900)], temporal instabilities in chemistry as observed in the Belousov-Zhabotinsky reaction [Belousov 1958] and mimicked by the Brusselator [Nicolis 1977], and the spatio-temporal Turing instability [Turing 1952]. Moving even further from equilibrium leads to the theory of chaos and complex systems which has been intensively treated at the end of the last century in particular by the Brussels' school [e.g. Prigogine 1980; Vidal 1991; Walgraef 1997; Gaspard 1998].

\section{Shortcomings}

Despite the considerable success and interest met by CIT, several shortcomings have been pointed out which have justified its extension and the formulation of other non-equilibrium theories. Let us list the most important criticisms of the classical approach to non-equilibrium thermodynamics.

1. By substituting the transport equations in the balance laws of mass, energy, and momentum, one obtains fields equations which are parabolic partial differential 
equations of the diffusion type; an example is the heat conduction equation in a rigid body

$$
\partial T / \partial t=\chi \nabla^{2} T
$$

where $\chi=\lambda / \rho c_{v}$ is the heat diffusivity, $c_{v}$ being the specific capacity. Solution of (2.12) implies that after application of temperature disturbance, the latter will be felt instantaneous and everywhere in the body, otherwise stated, temperature disturbance will move at infinite velocity across the body. This shortcoming was explicitly identified by Cattaneo [Cattaneo 1948] and was one of the first motivations to go beyond CIT. Propagation of signals with an infinite velocity is untenable from a purely conceptual physical point of view. However, from a practical point of view, the infinite speed of propagation is not an issue because in most situations, especially in low-frequency processes, the characteristic time is much longer than the transit time of the signals.

2. The local equilibrium hypothesis, which is the basic assumption underlying CIT, has been the subject of a great deal of controversy. According to it the space of state variables is the same as in equilibrium. However, it is conceivable that other variables, not relevant at equilibrium, may also influence the process. This is, for instance, the case with polymers of long molecular chains in which their configuration influences considerably their behavior. It is also so for super-fluids and super-conductors whose peculiar properties call for the introduction of additional variables.

3. The local equilibrium assumption implies large time and space scales as compared respectively to the collision times and mean free paths of particles. As a consequence, CIT is not appropriate for describing high-frequency phenomena such as ultrasound propagation and short-wavelength systems and processes, like nanostructures, shock waves, light and neutron scattering, fast phenomena as explosions, laser pulse heating and nuclear reactions.

4. According to generalized hydrodynamics, the phenomenological coefficients, like the heat diffusivity or the viscosity coefficient are frequency and wavelength dependent [Garcia-Colin 1984]. This is in contradiction with the local equilibrium hypothesis, which implies that these coefficients are frequency and wavelength independent.

5. As the flux-force relations are assumed to be linear, CIT cannot describe irreversible processes governed by non-linear phenomenological equations. Many phenomena, like chemical reactions or non-Newtonian flows, are among these classes of processes.

6. Another subject of controversy concerns the validity of the Onsager-Casimir reciprocal relations. Although they were originally derived by Onsager to be applicable only at the microscopic level for situations close to equilibrium, they are currently used by the followers of CIT at the macroscopic level and even far from equilibrium. There exists no theoretical argument supporting such an extrapolation. Moreover, in Onsager's point of view, the thermodynamic fluxes are defined as time derivatives of extensive thermodynamic variables, and the forces are the derivatives of the entropy with respect to the same state variables. These requirements are not fulfilled by thermodynamic fluxes like the heat flux or the pressure tensor and thermodynamic forces as the temperature gradient or the velocity gradient tensor.

7. Along the same lines, the important question has been raised of the unequivocal selection of fluxes and forces. One usually identifies the thermodynamic fluxes with the fluxes that appear in the balance laws of mass, momentum, and energy, while the forces are taken to be the conjugated terms in the bilinear expression of the entropy production. An alternative [de Groot 1962] is to consider as forces the gradient of intensive variables, such as temperature and velocity, and as fluxes 
the conjugate terms in the expression of entropy production. Unfortunately, such definitions are not applicable to chemical reactions. Another school of thought states that it makes no difference how the fluxes and forces are selected, as long as changing the frames of reference leave invariant the entropy production expression. But even if the reciprocal property of the phenomenological coefficients holds for a given choice of fluxes and forces, it may not hold for another selection [Coleman 1960; Wei 1974].

8. Minor criticisms concern the applications of the so-called Curie law according to which fluxes couple only with forces of the same tensorial order in isotropic materials. It is well known [Truesdell 1966, 1969] that such a conclusion is directly derivable from representation theorems of isotropic functions and there is no need to refer to the name of Curie to display this property. It is also important to keep in mind that "Curie's law" is not valid outside the linear regime even for isotropic bodies, a limitation sometimes forgotten by some authors.

To remedy the deficiencies of CIT, several formulations taking care of the above shortcomings, such as rational thermodynamics, internal variables theory, Hamiltonian structures, EIT have been developed: it is our purpose to focus on EIT whose early history (1953-1983) will be explored in the next sections. Comments on the other formalisms can be found in Section 8 .

\section{Early motivations and roots of EIT: generalized transport equations}

In Section 2, we have reviewed the classical transport laws of Fourier, Stokes and Newton. In these equations, the fluxes are proportional to the gradients of some quantities (temperature, velocity), evaluated at the same time. Thus, there exists an instantaneous relation between the gradient and the flux. This led to conceptual and practical problems, which have stimulated, since the times of Maxwell, the research on more general transport laws. One of the most obvious options was Maxwell's law for viscoelastic materials, in which a relaxation term given by a relaxation time multiplied by the time derivative of the pressure tensor $\boldsymbol{P}^{v}$ was added to Stokes law of viscous fluids. It follows that when the time-variation of $\boldsymbol{P}^{v}$ is small compared to the reciprocal of the relaxation time, the system behaves like a viscous fluid, but when its rate of change is important, the body behaves as an elastic solid. In this section we will comment on the need for such generalized equations.

\subsection{Cattaneo's work}

Cattaneo [Cattaneo 1948] was the first to try to solve the paradox of heat signals propagating at infinite speed. He suggested, for the problem of heat conduction in rigid bodies at rest, to replace Fourier's law by a non-stationary relation reflecting the property that the heat flux at a given time $t$ depends on the temperature gradient at an earlier time $\tau$ so that, after performing a Taylor expansion of $\nabla T(t-\tau)$, one obtains

$$
\boldsymbol{q}(t)=-\lambda \nabla T(t-\tau)=-\lambda\left(\nabla T-\tau \frac{\partial}{\partial t} \nabla T\right) .
$$

Unfortunately, this modification does not solve the problem. For $\boldsymbol{q}=0$, the temperature gradient tends exponentially toward infinity. Moreover, by replacing (3.1) in the energy balance law,

$$
\rho \frac{\partial u(T)}{\partial t}=-\nabla \cdot \boldsymbol{q}
$$


it is checked that the differential equation governing the behavior of the temperature is still parabolic. However, the paradox is easily solved by assuming

$$
\boldsymbol{q}(t+\tau)=-\lambda \nabla T(t),
$$

and, after a Taylor expansion of the heat flux, we get

$$
\tau \frac{\partial \boldsymbol{q}}{\partial t}+\boldsymbol{q}=-\lambda \nabla T
$$

which is nowadays known as the Cattaneo relation. Eliminating $\boldsymbol{q}$ between (3.4) and the energy conservation law (3.2), and using the equation of state $u(T)=c_{v} T$ with $c_{v}=\partial u / \partial T$ denoting the specific heat at constant volume, one is led to

$$
\tau \frac{\partial^{2} T}{\partial t^{2}}+\frac{\partial T}{\partial t}-\chi \nabla^{2} T=0
$$

with the assumption of constant values of $c_{v}$ and $\lambda$. This is a hyperbolic equation allowing for a finite velocity of propagation of thermal pulses, or high-frequency waves with a phase speed $v_{p h}$, given by:

$$
v_{p h}=\sqrt{\chi / \tau}
$$

Note that by setting $\tau=0$ in (3.6), which corresponds to Fourier's law, the velocity of propagation becomes infinite, as mentioned earlier. In a great number of problems, the relaxation time is very small, of the order of $10^{-10} \mathrm{~s}$ under normal conditions of temperature and pressure. This is the reason why generally in engineering science this effect is quantitatively too small to be taken into account. The velocity $v_{p h}$ is also called the second sound to be distinguished from the first sound which refers to a pressure wave moving with the velocity $\sqrt{(\partial p / \partial \rho)_{s}}$. The theory of solids predicts that $\chi / \tau=c_{0}^{2} / 3$ in the Debye approximation, with $c_{0}$ the sound velocity. After introducing this value in expression (3.6), it is found that the second sound is given by the constant value $v_{p h}=c_{0} / \sqrt{3}$. As a corollary, one obtains a quantitative estimate for the relaxation time in terms of measurable quantities, namely $\tau=3 \lambda / c_{v} c_{0}^{2}$. However, the result predicting that the second sound is constant is in conflict with experiments showing that it depends on temperature.

Interesting analyses of Cattaneo's relation are found in the works by Chester [Chester 1963, 1966]. Apparently unaware of Cattaneo's paper, which was written in Italian, Vernotte published a French version [Vernotte 1958] of Cattaneo's main results. During the same period, several attempts were made [Gurtin 1968; Luikov 1969; Bubnov 1976] to modify the heat conduction equation so as to predict thermal waves. Non-stationary expressions similar to Cattaneo's law (3.3) where the heat flux vector is replaced by the mechanical stress tensor were used to study magnetohydrodynamic flows [Wilhelm 1975].

\subsection{Grad's work}

The kinetic theory of gases, and more particularly the works of Grad have been a useful tool [Grad 1949, 1958] for the formulation of a non-equilibrium thermodynamics extending the scope of CIT. Grad was the first to elevate the fluxes to the rank of independent variables in his search to solve the Boltzmann equation in the case of an ideal or dilute monoatomic gas

$$
\frac{\partial f}{\partial t}+\boldsymbol{c} \cdot \frac{\partial f}{\partial \boldsymbol{r}}+\boldsymbol{F} \cdot \frac{\partial f}{\partial \boldsymbol{r}}=J(f f) .
$$


Here $f(\boldsymbol{r}, \boldsymbol{c}, t)$ denotes the one-particle distribution function giving, at time $t$, the number density of particles located in the interval $\boldsymbol{r}$ and $\boldsymbol{r}+d \boldsymbol{r}$ with velocity between $\boldsymbol{c}$ and $\boldsymbol{c}+d \boldsymbol{c}, \boldsymbol{F}$ is the external force and $J(f f)$ the collision integral. The connection between kinetic and continuum theories is made through the construction of moments of the distribution function. Such moments are, for instance, the five conserved quantities

$$
\begin{aligned}
\rho & =m \int f d \boldsymbol{c}(\text { mass }), \rho \boldsymbol{v}=m \int \boldsymbol{c} f d \boldsymbol{c}(\text { momentum }), \\
\rho u & =\frac{1}{2} m \int C^{2} f d \boldsymbol{c}(\text { internal energy }),
\end{aligned}
$$

and the energy and momentum fluxes defined, respectively, by

$$
\begin{aligned}
\boldsymbol{q} & =\frac{1}{2} m \int C^{2} \boldsymbol{C} f d \boldsymbol{c}, \\
\boldsymbol{P} & =m \int \boldsymbol{C} \boldsymbol{C} f d \boldsymbol{c}
\end{aligned}
$$

with $\boldsymbol{C}$ standing for the relative velocity $\boldsymbol{C}=\boldsymbol{c}-\boldsymbol{v}$ with respect to the mean velocity $\boldsymbol{v}$ and $m$ for the mass of the particles. Grad's method consists in expanding the non-equilibrium distribution function $f(\boldsymbol{r}, \boldsymbol{c}, t)$ in terms of a complete set of Hermite polynomials, around the local Maxwellian equilibrium distribution $f_{e q}(\rho, u)$. The coefficients in this expansion are identified as the five conserved densities plus higher-order moments, their time evolution is governed by an infinite set of coupled differential equations which, when truncated at an appropriate level of moments, can be solved. In the thirteen-moment approximation, taken here as an illustration, the distribution function is not only a function of the conserved variables through the Maxwellian distribution function $f_{e q}$ but also of the heat flux and the traceless deviatoric pressure tensor, namely

$$
f=f_{e q}\left[1+\frac{m}{2 k_{B} T} \stackrel{0}{\boldsymbol{C}}: \stackrel{0}{\boldsymbol{P}^{v}}+\frac{m}{5 p k_{B}^{2} T^{2}}\left(m C^{2}-5 k_{B} T\right) \boldsymbol{C} \cdot \boldsymbol{q}\right],
$$

a colon stands for the double scalar product. Expression (3.11) implies that the nonequilibrium entropy density defined classically by

$$
\rho s=-k_{B} \int f \ln f d \boldsymbol{c},
$$

is not only a function of the conserved variables $\rho$ and $u$ but depends in addition on the fluxes $\boldsymbol{q}$ and $\boldsymbol{P}^{0 v}$ so that one is allowed to write:

$$
s=s\left(\rho, u, \boldsymbol{q}, \boldsymbol{P}^{0 v}\right) .
$$

The enlargement of the space of state variables appears therefore as a direct consequence of Grad's method, and relation (3.13) expressing the entropy function in terms of the 13 moments can be viewed as the fundamental postulate of the macroscopic formulation of EIT as it confirms the status of $\boldsymbol{q}$ and $\stackrel{0}{\boldsymbol{P}^{v}}$ as state variables. This procedure has been generalized to include 26 and more moments [Velasco 1993; Müller 1993], but the problem with higher order moments is their identification at the macroscopic level. However, Grad's objective was not to construct a thermodynamic theory generalizing CIT so that, in that perspective, his contribution passed rather unnoticed. 


\section{A short conceptual presentation of extended irreversible thermodynamics}

Before presenting the historical evolution, we provide here a short introduction to the essential concepts of extended thermodynamics. For pedagogical reasons, the analysis of the foundations underlying the early versions of EIT will be given here by using as a case-study the rigid heat conductor at rest ( $\rho=$ const., $\boldsymbol{v}=0$ so that the material time derivative may be identified with the partial time derivative: $d / d t=\partial / \partial t$ ). The generalization to more complicated systems such as fluids, mixtures, suspensions, porous media, and others has been dealt with in detail in numerous publications. Recall that one of the motivations behind EIT is to remove the possibility of waves propagating at infinite velocity. This can be accomplished by assuming that the fluxes, here the flux of energy, are taken as state variables so that the space $\boldsymbol{V}$ of state variables will be formed by the union of the (slow and conserved) classical variables $\boldsymbol{C}$ and the (fast and non-conserved) flux variables $\boldsymbol{F}$. In the problem of heat transport, the single conserved variable is the internal energy $u$ and the heat flux $\boldsymbol{q}$ is the nonconserved flux variable so that the space of state variables $\boldsymbol{V}$ consists of the pair $u$, $\boldsymbol{q}$. The corner stone of EIT is to assume the existence of an entropy function $s$ that depends on the whole set $\boldsymbol{V}$ of variables, here $u$ and $\boldsymbol{q}$, and whose rate of production is positive definite; in addition, it is assumed to be a concave function of the variables to guarantee stability of the equilibrium state. In differential form, one has

$$
\left.\left.d s(u, \boldsymbol{q})=\frac{\partial s}{\partial u}\right)_{\boldsymbol{q}} d u+\frac{\partial s}{\partial \boldsymbol{q}}\right)_{u} \cdot d \boldsymbol{q}
$$

wherein a sub-index means that the corresponding quantity remains constant. Set $\partial s / \partial u)_{\boldsymbol{q}}=\theta^{-1}(u, \boldsymbol{q})$ with $\theta(u, \boldsymbol{q})$ being defined as the absolute non-equilibrium temperature and $\partial s / \partial \boldsymbol{q})_{u}=\alpha(u, \boldsymbol{q}) / \rho$, with the factor $1 / \rho$ being introduced for convenience, $\boldsymbol{\alpha}(u, \boldsymbol{q})$ is a quantity depending in general on $u$ and $\boldsymbol{q}$, to be given by a constitutive relation. In terms of the material time derivative, expression (4.1) is then given the form

$$
\frac{d}{d t} s(u, \boldsymbol{q})=\frac{1}{\theta} \frac{d u}{d t}+\frac{\boldsymbol{\alpha}}{\rho} \cdot \frac{d \boldsymbol{q}}{d t} .
$$

The non-equilibrium temperature $\theta(u, \boldsymbol{q})$ should not be confused with the (local) equilibrium temperature. A Taylor expansion of $\theta(u, \boldsymbol{q})$ around $\boldsymbol{q}=0$ results in

$$
\theta(u, \boldsymbol{q})=T(u)+\gamma(u) q^{2}+\ldots
$$

where $T(u)$ stands for $\theta(u, 0)$ and represents the equilibrium temperature which is $\boldsymbol{q}$-independent, $\gamma(u)$ is a phenomenological coefficient; it is worth to stress that $\theta$ reduces to $T$ when second order contributions in $q^{2}$ are omitted. For a detailed review and discussion on the definitions of non-equilibrium temperature, see the works of Luzzi et al. and Casas-Vazquez and Jou [Luzzi 1997; Casas-Vazquez 2003].

From the isotropy property of constitutive equations, one may write $\boldsymbol{\alpha}=a\left(u, q^{2}\right) \boldsymbol{q}$ where $a$ is a scalar function of $u$ and $q^{2}$. Substituting this expression in (4.1) and making use of the energy conservation law (3.2), it is seen that $d s / d t$ can be cast in the form

$$
\rho \frac{d s}{d t}=-\nabla \cdot \frac{\boldsymbol{q}}{\theta}+\boldsymbol{q} \cdot\left(\nabla \theta^{-1}+a \frac{d \boldsymbol{q}}{d t}\right) .
$$


Comparing with the general balance equation for entropy expressed by (2.2), we read off the identifications

$$
\begin{aligned}
\boldsymbol{J}^{s} & =\boldsymbol{q} / \theta \quad \text { (entropy flux), } \\
\sigma^{s} & =\boldsymbol{q} \cdot\left(\nabla \theta^{-1}+a \frac{d \boldsymbol{q}}{d t}\right) \geqslant 0 \quad \text { (rate of entropy production per unit volume). }
\end{aligned}
$$

Assuming in (4.6) a linear relation $\boldsymbol{q}=L \boldsymbol{X}$ between the flux $\boldsymbol{q}$ and the force $\boldsymbol{X}$, represented by the two terms in the parentheses, one finds

$$
\boldsymbol{q}=L\left(-\frac{1}{\theta^{2}} \nabla \theta+a \frac{d \boldsymbol{q}}{d t}\right) .
$$

With the identifications $L a=\tau$ (relaxation time) and $L / \theta^{2}=\lambda$ (heat conductivity), one recovers Cattaneo's law

$$
\tau \frac{d \boldsymbol{q}}{d t}=-\boldsymbol{q}-\lambda \nabla \theta
$$

Some considerations about the signs of the various phenomenological coefficients are in order: substitution of the linear flux-force law $\boldsymbol{q}=L \boldsymbol{X}$ in (4.5) leads to

$$
\sigma^{s}=\boldsymbol{q} \cdot \boldsymbol{q} / L \geqslant 0
$$

and therefore $L>0$ whence $\lambda>0$; furthermore, integration of the Gibbs relation (4.2) up to second order terms in $q$ yields

$$
s(u, \boldsymbol{q})=s_{e q}(u)+(1 / 2)(a / \rho) q^{2},
$$

from which follows $a<0$ to satisfy the property that $s$ is maximal at (local) equilibrium. By combining $a<0$ and $L>0$, it is concluded that $\tau>0$, indicating that the relaxation time is indeed a positive quantity, as it should. The above results exhibit the main achievements of EIT in the case of relaxational heat transport.

To explore further the physical contents of Cattaneo's relation (4.8), let us write its solution as

$$
\boldsymbol{q}(t)=\int_{-\infty}^{0} \frac{\lambda}{\tau} \exp \left(-\frac{t-t^{\prime}}{\tau}\right) \nabla \theta\left(t^{\prime}\right) d t^{\prime},
$$

which illustrates the property that the heat flux $\boldsymbol{q}(t)$ possesses memory with an exponential memory decay. It was shown in Section 3 that elimination of $\boldsymbol{q}$ between the energy and Cattaneo's law yields the telegrapher equation (3.5). For short time scales $t / \tau \ll 1$, the first term of equation (3.5) is dominant, so that it reduces to

$$
\tau \frac{\partial^{2} T}{\partial t^{2}}=\chi \nabla^{2} T
$$

which is the classical wave equation describing a reversible process as it is invariant with respect to time reversal $t \rightarrow-t$. In contrast, for long time scales $t / \tau \gg 1$, the first term of (3.5) is negligible and one recovers the heat diffusion equation (2.12), which is not invariant when $t$ is changed into $-t$ as it is related to an irreversible process. To summarize, at short times the telegrapher transport equation (3.5) is reversible and heat propagates with a finite speed (which may be interpreted microscopically as a ballistic motion of the particles before experiencing a collision), whereas at longer times the process becomes irreversible and heat is diffused throughout the system. In that respect, the telegrapher equation is more general than Fourier's law, as it describes the transition from the reversible to the irreversible behavior, with $\tau$ interpreted as the characteristic time for the crossover between ballistic motion and the 
onset of diffusion. In the context of the theory of deterministic chaos, $\tau$ corresponds to the Lyapunov time beyond which predictivity is lost [Nicolis 1989].

The above results are easily generalized to the study of a thermo-viscous fluid. Taking for the entropy

$$
s=s\left(\rho, u, \boldsymbol{q}, \stackrel{0}{\boldsymbol{P}^{v}}, p^{v}\right),
$$

the corresponding Gibbs equation takes the form

$$
\frac{d s}{d t}=\theta^{-1} \frac{d u}{d t}+\pi \theta^{-1} \frac{d\left(\rho^{-1}\right)}{d t}+a_{1} \boldsymbol{q} \cdot \frac{d \boldsymbol{q}}{d t}+a_{2}{\stackrel{0}{\boldsymbol{P}^{v}}}^{v}: \frac{d \boldsymbol{P}^{v}}{d t}+a_{3} p^{v} \frac{d p^{v}}{d t},
$$

where $\theta$ and $\pi$ stand for the (non-equilibrium flux-dependent) temperature and (nonequilibrium flux-dependent) pressure, whereas the derivatives of $s$ with respect to the fluxes have been identified as

$$
\partial s / \partial \boldsymbol{q}=a_{1} \boldsymbol{q}, \partial s / \partial \boldsymbol{P}^{v}=a_{2}{\stackrel{0}{\boldsymbol{P}^{v}}}^{v} \partial s / \partial p^{v}=a_{3} p^{v},
$$

respectively. Neglecting non-linear contributions in the fluxes so that it is justified to replace $\theta$ by $T$, making use of the balance equations of mass and energy and following the same procedure as in the case of heat conduction, one is led to the next linear evolution equations,

$$
\begin{aligned}
\tau_{1} \frac{d \boldsymbol{q}}{d t} & =-(\boldsymbol{q}+\lambda \nabla T), \\
\tau_{0} \frac{d p^{v}}{d t} & =-\left(p^{v}+\zeta \nabla \cdot \boldsymbol{v}\right), \\
\tau_{2} \frac{d \boldsymbol{P}^{0 v}}{d t} & =-\left(\boldsymbol{P}^{0 v}+2 \eta \boldsymbol{V}^{0}\right),
\end{aligned}
$$

where $\tau_{1}, \tau_{0}, \tau_{2}$ are relaxation times. When these times are negligible, the set $(4.15)-$ (4.17) reduces to the Navier-Fourier-Stokes relations (2.9)-(2.11). One recognizes Cattaneo's and Maxwell's equations in expressions (4.15) and (4.17), respectively. Although the linear equations (4.13)-(4.15) predict that disturbances move at finite speed, there is no guarantee that the same property holds for non-linear systems.

The above results are easily generalized by changing the identification of $\boldsymbol{J}^{\boldsymbol{s}}$, to

$$
\boldsymbol{J}^{s}=\boldsymbol{q} / T+\beta^{\prime} p^{v} \boldsymbol{q}+\beta^{\prime \prime} \boldsymbol{P}^{v} \cdot \boldsymbol{q}
$$

which is derived straightforwardly from the representation theorems of isotropic functions and where $\beta^{\prime}$ and $\beta^{\prime \prime}$ are phenomenological coefficients. Relation (4.18) is important because it represents an example wherein the colinearity between $\boldsymbol{J}^{s}$ and $\boldsymbol{q}$ as expressed by (4.5) is given up.

Writing the entropy production in the form

$$
\sigma^{s}=\rho d s / d t+\nabla \cdot J^{s}
$$

and making use of (4.14) and (4.18), one obtains for $\sigma^{s}$ a bilinear relation in the fluxes and generalized forces containing time derivatives and spatial gradients. Assuming linear flux-force relations leads to the following set of evolution equations (Lebon 2008a; Jou 1983, 1993):

$$
\begin{aligned}
\tau_{1} \frac{d \boldsymbol{q}}{d t} & =-(\boldsymbol{q}+\lambda \nabla T)+\beta^{\prime \prime} \lambda T^{2} \nabla \cdot \boldsymbol{P}^{v}+\beta^{\prime} \lambda T^{2} \nabla p^{v} \\
\tau_{0} \frac{d p}{d t^{v}} & =-\left(p^{v}+\zeta \nabla \cdot \boldsymbol{v}\right)+\beta^{\prime} \zeta T \nabla \cdot \boldsymbol{q} \\
\tau_{2} \frac{d \boldsymbol{P}^{0 v}}{d t} & =-\left(\boldsymbol{P}^{0 v}+2 \eta \boldsymbol{V}^{0}\right)+2 \beta^{\prime \prime} \eta T\left(\nabla^{0} \boldsymbol{q}\right)^{s}
\end{aligned}
$$


which are more general then Cattaneo's and Maxwell's relations by the presence of non-local terms in the gradients of $p^{v}, \boldsymbol{q}$ and $\stackrel{0}{\boldsymbol{P}}^{v}$. These are related to contributions coming from the entropy flux, whereas those involving the relaxation effects are associated to the non-classical terms in the Gibbs equation (4.14). These results show that generalized transport equations and extended forms of the entropy and entropy flux are closely related to each other.

Although the first formulations of EIT are satisfactory from several physical aspects by avoiding the paradox of signals propagating at infinite velocity and embracing rather general evolution equations like (4.20)-(4.22), they present some shortcomings. First, hyperbolicity is not universally guaranteed and requires, in particular, that some bounds be put on the field variables [Jou 1993; Müller 1998]. Moreover, it was shown that for shock waves with a Mach number beyond some critical value, the shock structure is lost in contradiction to the Navier-Fourier-Stokes equations where the shock is preserved whatever the value of the Mach number. In the 13-moment approximation, which is equivalent to taking $\boldsymbol{q}$ and the traceless $\stackrel{0}{\boldsymbol{P}}^{v}$ as variables, the critical Mach number [Ruggeri 1993; Müller 1998] is $\mathrm{Ma}=1.65$ and this value increases only slowly when the number of moments is increased [Weiss 1990]. Another criticism concerns expression (4.18) of the entropy flux. The additional terms (second and third) do not receive a solid justification unless they may be supported by kinetic or statistical mechanical arguments. Moreover, by comparing the theoretical results of wave speed and absorption coefficients with experimental data, one observes a satisfactory agreement only for values of $\omega \tau<2$ [Anile 1984; Lebon 1989]. In addition, the above relations contain non-objective time derivatives and therefore do not comply with the criterion of material frame indifference [Noll 1965]. From a more fundamental point of view, the approach was criticized by van Kampen for whom the use of fast variables, like the fluxes, is not justified [van Kampen 1987] because only slow variables should enter into a macroscopic description. Van Kampen's arguments are based on Chapman-Enskog's treatment where higher-order fluxes are not selected as variables, in contrast with Grad's method. However, Chapman-Enskog's iterations, as it is actually well-known, lead to instabilities, e.g. [Struchtrup 2005], which is not the case with Grad's approach. More generally, for van Kampen and others, one has the microscopic world with the kinetic theory and one has the macroscopic level with hydrodynamics, but no mesoscopic level between them.

It was also argued that if the relaxation times of all the higher-order variables are of the same order, it is not sufficient to add the single first-order fluxes, but the fluxes of all higher orders should be incorporated into the description [Garcia-Colin 1995]. To deal with this objection, a hierarchy of equations for higher-order fluxes up to infinity has been introduced, and, instead of truncating them at given order of approximation, which is the common practice, some asymptotic expressions are derived, leading to the definition of renormalized flux relaxation times. The goal of this procedure is to take into account all the higher-order fluxes via these renormalized relaxation times in such a way that only the first order flux, say the usual heat flux or/and the viscous pressure tensor are needed in the analysis [Jou 1993].

\section{The several independent formulations of EIT}

Under the label of EIT, one finds a great variety of approaches to cope with the difficulties discussed above. This is a particular illustration of a theory proposed by different researchers, who start from different motivations and on different grounds, often ignorant of each other's contributions, probably because of the long interval of time elapsed between several consecutive proposals and the specific aims and ego 
of each researcher. The fact that the theory has been put forward independently on several occasions is not strange, as its ideas seem quite reasonable and conceptually worth to be explored.

\subsection{Machlup and Onsager (1953): A formal kinetic extension of constitutive equations}

To our knowledge, the first reference to a generalized entropy depending on the fluxes and related to relaxational constitutive equations was formulated by Machlup and Onsager, in 1953, then at Yale University, as the second part of their well-known analysis of fluctuations and irreversible processes. These authors refer in the subtitle of their paper to "systems with kinetic energy" to stress this dependence. They work in the abstract general framework set out by Onsager in 1931, wherein the fluxes are expressed as the time derivatives (denoted here by a upper dot) $\dot{\alpha}_{i}$ of the variables $\alpha_{i}$. In their paper of 1953, Machlup and Onsager wrote for the global entropy $S$.

$$
S\left(\alpha_{i}, \dot{\alpha}_{i}\right)=S_{0}-(1 / 2) \sum_{i, j} s_{i j} \alpha_{i} \alpha_{j}-(1 / 2) \sum_{i, j} m_{i j} \dot{\alpha}_{i} \dot{\alpha}_{j}
$$

with $S_{0}$ a reference value of the entropy; $s_{i j}, m_{i j}$ are coefficients related to the second derivatives of the entropy with respect to $\alpha_{i} \alpha_{j}$ and $\dot{\alpha}_{i} \dot{\alpha}_{j}$, respectively. According to Machlup and Onsager, the evolution equations will be given the form

$$
\sum_{j} m_{i j} \ddot{\alpha}_{j}+\sum_{j} R_{i j} \dot{\alpha}_{j}-\partial S / \partial \alpha_{i}=0 .
$$

For $m_{i j}=0$, the second-order time derivative disappears from (5.2), as well as the contribution of the fluxes to the entropy, and both (5.1) and (5.2) reduce to

$$
\begin{aligned}
S & =S_{0}-(1 / 2), \sum_{i, j} s_{i j} \alpha_{i} \alpha_{j} \\
R_{i j} \dot{\alpha}_{j} & =\frac{\partial S}{\partial \alpha_{i}}=X_{i},
\end{aligned}
$$

with $X_{i}$ standing for the "thermodynamic force". Machlup and Onsager dubbed the contribution of the fluxes to (5.1) as "kinetic contribution" due to the "inertia" of the system. Starting from the above results, Machlup and Onsager discussed a set of linear, second-order, stochastic processes, analyzed the fluctuations and the reciprocal relations amongst the coefficients, as well as the "fluctuation paths" of the fluctuations. They pointed out that in the generalized case, the "thermodynamic forces" should be written as:

$$
X_{i}=\frac{\partial S}{\partial \alpha_{i}}+\frac{d}{d t} \frac{\partial S}{\partial \dot{\alpha}_{i}}
$$

emphasizing the analogy between the second term of this sum with d'Alembert's force in mechanics and Lagrange equations for non-conservative systems.

However, Machlup and Onsager's ideas did not percolate through macroscopic thermodynamics, because the authors turned their attention to other problems (biophysical ones for Machlup, quantum aspects of vorticity in superfluids for Onsager). Although the essential ideas of extended thermodynamics were already rooted in Machlup and Onsager's paper, it did not lead to fruitful developments and was forgotten and remained unmentioned in the great majority of works on EIT. 


\subsection{Nettleton (1959-1960): Heat flux and viscoelasticity in liquids}

The next researcher to explore topics related to extended thermodynamics was Nettleton, apparently unaware of the previous proposal by Machlup and Onsager. Nettleton studied relaxation equations for viscoelastic liquids [Nettleton 1959] and for thermal conductors [Nettleton 1960]. For the first time, it was proved that Cattaneo's and Maxwell's viscoelastic relations follow directly from thermodynamics, provided the classical Gibbs equation is supplemented by extra contributions in the fluxes. It was the merit of Nettleton to select fluxes as independent variables at very short times, high frequencies, and non-equilibrium states within a thermodynamic context. Identifying the heat flux $\boldsymbol{q}$ with an internal ordering parameter $\boldsymbol{\xi}$, Nettleton wrote the Gibbs' equation in a form reminiscent of that used to describe chemical reactions,

$$
T d s=d u-p d v+\Phi \cdot d \boldsymbol{\xi}
$$

with $\Phi$ the variable conjugated to $\boldsymbol{\xi}$, identified as $\Phi=-\boldsymbol{\xi} / \tau \omega$, where $\omega$ is a phenomenological parameter to be shown to be related to the reciprocal of the heat conductivity $\lambda$. Although Nettleton did not derive explicitly Cattaneo's equation (referred to by him as Vernotte's equation), he assumed that $\boldsymbol{\xi}$ obeys a relaxation equation of the Cattaneo type

$$
d \boldsymbol{\xi} / d t=-\boldsymbol{\xi} / \tau-(\lambda / \tau) \nabla T
$$

Nettleton was especially interested in heat transport in liquids, where the heat flux may be split into two independent contributions, one of them related to sound waves and the other one to diffusing particles. He derived on a microscopic basis the various non-equilibrium terms in the entropy and he used the equality of the second-order crossed derivatives (analogous to the Maxwell relation of usual equilibrium thermodynamics) to relate information on crossed effects between the mentioned contributions to the heat flux.

These relevant contributions of Nettleton, supplemented by deep microscopic insights based on kinetic theory of liquids, did not receive the interest they deserved and were practically ignored in the thermodynamic literature for twenty years. A possible reason was that these papers were not very accessible because of the complexity of the system being studied. Moreover, instead of writing the Gibbs equation directly in terms of the fluxes, Nettleton viewed the fluxes as internal variables. Instead of presenting his work as the possible foundation of a new theory, he focused his attention on particular problems, as if the formalism was specifically tailored for them, instead of representing a contribution towards a more general formalism. Nettleton's ideas were brought back to life after he presented his work at the Bellaterra conference held in 1983 [Nettleton 1984]. In collaboration with Sobolev [Nettleton 1995], he wrote two review papers on the applications of extended thermodynamics.

\subsection{Müller (1966-1983): Kinetic theory of gases and generalized theory}

Probably unaware of the ideas set forth by Nettleton, Ingo Müller in his Ph.D. Thesis [Müller 1966] at the Technical High School at Aachen, under the supervision of Meixner, put EIT in a more structured and systematic form. At this time, Meixner played an important role in the development of non-equilibrium thermodynamics not only by his criticisms of contemporary theories but principally by the formulation of an original approach [Meixner 1968] referred to as "entropy free thermodynamics". Meixner's motivation was the observation that entropy can only receive an accurate and univocal definition in equilibrium situations. However, because of the competition with the Brussels school headed by Prigogine and Rational Thermodynamics (see Sect. 8.1), Meixner's theory did not meet the credit that it deserved. 
Let us go back to Müller's work. In his basic paper of 1967 [Müller 1967], he follows the lines of thought of classical irreversible thermodynamics but with two important differences; first, the entropy $s$ is allowed to depend on the fluxes, and second, non-collinearity between entropy flux and heat flux is postulated. In the case of a thermo-viscous fluid, the entropy $s$ is assumed to be a function of the same variables as in (4.13) with the entropy flux given by (4.18). Taking the time derivative of expression (4.13) and making use of the balance equations for density and internal energy, one identifies the entropy production $\sigma^{s}$ as a bilinear structure of fluxes and forces. Assuming linear relations between fluxes and forces, one obtains back the set $(4.20)-(4.22)$.

Later on, Müller introduced the notion of coldness as a generalization of the absolute equilibrium temperature [Müller 1970, 1971]. He proposed to substitute the classical expression (4.5) of the entropy flux by a more general expression

$$
\boldsymbol{J}^{s}=\Lambda\left(T_{e m}, \frac{d T_{e m}}{d t}\right) \boldsymbol{q},
$$

where $T_{e m}$ designates the empirical temperature that can be measured by a thermometer, $\Lambda$ was dubbed "coldness", to be given by a constitutive equation depending on $T_{e m}$ and $d T_{e m} / d t$; its existence rests on the postulate that there exist "ideal walls" across which the temperature is continuous at all times, and that at such walls, the normal components of the heat flux and the entropy flux are continuous. It was proved that $\Lambda$ is the same function for all materials, or, in Müller's terminology, that $\Lambda$ is a universal function, which, in equilibrium is the inverse of the absolute temperature: $\Lambda\left(T_{\text {em }}, 0\right)=1 / T$. In Müllers's view, the absolute temperature is not a primitive quantity but rather a derived one which depends on the empirical temperature. The notion of coldness was criticized by Meixner who proved that $\Lambda$ is a universal function, for a restricted class of materials only, quoting him [Meixner 1974], "In the super class $\Lambda$ is no longer a universal function and in non-equilibrium, it does not seem appropriate to consider $T_{e m}$ as a temperature". Nevertheless, considering the entropy and the heat fluxes through an interface between two systems (say, a thermometer and a system) is convenient, as it is reminiscent of the contact between the system and the thermometer in measuring temperature. Although the existence of ideal interfaces is an interesting theoretical concept, in general the interfaces between different materials are not ideal, but exhibit so-called thermal boundary resistance, which implies not only a discontinuity of temperature through the surface, but concomitantly a discontinuity of the entropy flux, due to entropy production across the wall. Similar situations are not found in equilibrium thermodynamics, where the thermal contact is universal, i.e. independent of the degrees of freedom involved. This dependence on thermal contact implies that the extension of the zeroth principle of thermodynamics to non-equilibrium situations remains a delicate matter [Muschik 1977; Casas-Vazquez 2003]. Finally, one must be sure that only heat flux is exchanged between the two systems separated by the interface, at the exclusion of other quantities like matter, or dislocations. In view of the above and other criticisms, the idea of coldness was later abandoned by Müller and his collaborators. A good summary of Müller's work during this period can be found in the review article by Hutter [1977].

After his Ph.D. thesis, Müller moved to the USA and became familiar with rational thermodynamics [Coleman 1963, 1964]. For some years he abandoned extended thermodynamics, but in 1983 he revisited it by using his knowledge of the techniques of rational thermodynamics, but without adhering to the Clausius-Duhem inequality with the entropy flux identified as $\boldsymbol{q} / T$. In a paper co-authored with Liu, Müller presented a new approach of extended thermodynamics [Liu 1983] using the methods of rational thermodynamics instead of those of classical irreversible thermodynamics. The ideas developed in this article were made more explicit and amplified to a 
monograph with Ruggeri, from Bologna University, entitled "Extended Thermodynamics" (1993); a second enlarged and completely revised version entitled "Rational Extended Thermodynamics" was published in 1998. Müller and Ruggeri have developed a mathematically elegant theory, however, applications to complex systems, as for instance real gases or macromolecular solutions, remain not straightforward. One reason is that for such systems, the heat flux and the viscous pressure tensor are no longer single physical entities with one single relaxation time, but a sum of several contributions with crossed effects, related to particle interactions and macromolecular internal structures.

Inspired by the developments of rational thermodynamics, where the entropy inequality is regarded as a constraint to the constitutive equations, EIT is considered by Müller as the combination of the classical balance equations of mass, momentum, energy, and entropy with the time evolution equations of higher-order flux quantities: the Gibbs equation is now a derived relation rather than a postulated statement. In addition, the evolution equations of mass, momentum, energy and higher order moments are viewed as providing constraints to the field variables $u_{\alpha}$ (say $\rho, \boldsymbol{v}, u$ and higher order moments). Following Liu and Müller, the entropy inequality written in the form [Liu 1972, 1983]

$$
\begin{aligned}
\rho \frac{\partial s}{\partial t} & +\nabla \cdot \boldsymbol{J}^{s}+\boldsymbol{\Lambda}_{m}(\text { mass balance })+\boldsymbol{\Lambda}_{v} \cdot(\text { momentum balance })+\Lambda_{u}(\text { energy balance }) \\
& +\boldsymbol{\Lambda}_{i j k \ldots}(\text { higher-order balance equations }) \geqslant 0
\end{aligned}
$$

must hold for all the set of variables, the $\Lambda_{i}$ 's are denoting here the Lagrange multipliers, which are introduced to eliminate the constraints set by the balance equations. At the end of the procedure, the $\Lambda_{i}$ 's will be identified in terms of the field variables. In addition, it is required that $s$ is a concave function, $\partial^{2} s / \partial u_{\alpha} \partial u_{\beta} \leqslant 0$, to guarantee stability of the equilibrium solutions and that the set of field equations are symmetric hyperbolic. This ensures not only finite speeds but also that, mathematically, the Cauchy problem is well-posed: uniqueness, existence, and continuous dependence of solutions are guaranteed. Moreover, in order to study shock waves, it is important to reformulate the field equations such that they exhibit a conservative form. The characteristic speeds, and, in particular, the maximum one, which represents the pulse speed, are easily calculated from the characteristic equation. In the case of a monatomic gas, the result depends on the number of moments selected for the description; the problem is that by increasing the number $n$ of moments [Weiss 1990], the speed pulse increases with $n$ without upper bound. This is a rather disappointing result as one of the main motivations for the theory was to find a finite speed of signals. However, this is not completely surprising as Boltzmann's equation allows for a non-vanishing probability of particles moving at any chosen speed. In Müller's approach, the problem of a non-equilibrium temperature is scarcely discussed; indeed, because the scheme is deeply rooted in the kinetic theory, the temperature is identified with the energy density, namely $u=(3 / 2) k_{B} T / m$ for a monatomic gas.

\subsection{G. Lebon (1973-1978), D. Jou et al. (1979-1980): From fast processes to thermodynamics of steady states}

In parallel to Müller, Lebon and Lambermont at Liège University, studied the problem of propagation of heat signals in rigid thermo-elastic bodies and viscous heat conducting fluids in the framework of EIT [Lambermont 1973; Lebon 1973, 1978]. Works by Lebon and Lambermont were put into a more general and structured form in two papers written in collaboration with Jou and Casas-Vázquez, from the Universitat 
Autònoma de Barcelona [Jou 1979; Lebon 1980a]. The main objective of these two contributions was to go beyond the local equilibrium hypothesis by raising the fluxes to the status of truly independent variables; their relaxation constitutive equations, containing the time derivative of the fluxes, were not seen as a correction to the classical constitutive equations, but as evolution equations for the fluxes. Non-equilibrium entropy $s_{n e q}$, is not only a function of the classical conserved variables, as energy, but depends in addition on the dissipative fluxes, like the energy flux. For instance in the case of heat conduction, at the lowest order in $q^{2}$, one has

$$
s_{n e q}(u, \boldsymbol{q})=s_{e q}(u)-\left(\tau / 2 \lambda T^{2}\right) q^{2}
$$

with $s_{e q}(u)$ standing for the local equilibrium entropy, it is worth to stress that the coefficient in front of $q^{2}$ is expressed in terms of well-identified physical quantities as the heat conductivity $\lambda$ and the relaxation time $\tau$. This formulation gave a more definite and transparent status to the extended entropy, which previously could appear as only a formal proposal without a clear physical interpretation. Expression (5.10) of the entropy was seen to coincide with that obtained from the kinetic theory, both in Grad's and (for steady or slowly changing states) in Chapman-Enskog's treatments.

Extended entropy plays also a significant role in the description of fluctuations. For simplicity, we will restrict ourselves to fluctuations of the heat flux. Denoting by $\delta \boldsymbol{q}$ the fluctuation of $\boldsymbol{q}$ around its equilibrium value and substituting expression (5.10) of entropy in Einstein's relation [Landau 1980] for the probability of fluctuations $W$, one obtains

$$
W \approx \exp \frac{1}{2} \frac{\delta^{2} s}{k_{B}} \approx \exp \left(-\frac{\tau}{\rho \lambda T^{2}} \delta \boldsymbol{q} \cdot \delta \boldsymbol{q}\right) .
$$

From this result follows that the second moment of fluctuations is given by [Landau 1980]

$$
\langle\delta \boldsymbol{q} \delta \boldsymbol{q}\rangle=\left(k_{B} \rho T^{2} \lambda / \tau\right) \boldsymbol{I},
$$

where the brackets stand for average values. Relation (5.12) emphasizes the link between the dissipative coefficient $\lambda$ and the fluctuation of the heat flux and is an illustration of the well-known fluctuation-dissipation theorem, e.g. [Reichl 1980]: it provides a supplementary argument in favor of the physical consistency of the generalized entropy.

Once the expression of entropy is known, there is no difficulty in deriving the corresponding equations of state that are directly obtained by simple derivation with respect to the state variables. This is particularly relevant in the study of nonequilibrium steady states. This may seem surprising, as EIT found its early motivations in the need to cope with rapidly changing states. However, there is no contradiction: indeed, when the energy fluxes are important, energy is entering and leaving the system at a fast rate, and the system becomes unable to thermalize during the short transit period that the energy spends in the system. Therefore, temporal arguments are important even in non-equilibrium steady states. Like in Müller's theory, the expression of the entropy flux $\boldsymbol{J}^{s}$ will contain supplementary contributions in the fluxes besides the classical term $\boldsymbol{q} / T$ and the rate of entropy production $\sigma^{s}$ is assumed the be positive definite, at each point in space and at each instant of time, this property is exploited to put restrictions on the sign of the various phenomenological coefficients appearing in the evolution equations, as the heat conductivity, the bulk and shear viscosities.

Furthermore, some particular classes of problems like matter diffusion [Lebon 1980a], micropolar media [Rubí 1980], and charged particles systems [Jou 1982] received a specific treatment. 


\subsection{Gyarmati (1977): Wave approach to non-equilibrium thermodynamics}

Akin to the above treatments, Gyarmati, at the Polytechnical University of Budapest, proposed a so-called wave approach of irreversible processes [Gyarmati 1977]. The latter was presented by the author as a generalization, to continuous media of the Onsager-Machlup theory with the entropy given by

$$
s=s\left(\alpha_{i}\right)-\frac{1}{2} \sum_{i, j} m_{i j} J_{i} J_{j},
$$

where the $J_{i}$ are velocity-type variables, while $m_{i j}$ are tensors supposed to be symmetric and non-negative. The $J_{i}^{\prime} s$ are not the time derivatives of the state variables $\alpha_{i^{\prime}}$ but defined through balance equations of the general form

$$
\frac{d \alpha_{i}}{d t}=-\nabla \cdot \boldsymbol{J}_{i}+\sigma_{i}
$$

with $\sigma_{i}$ the source density of the $\alpha_{i}$. Gyarmati introduces also generalized thermodynamic forces $X i$, dependent not only on the gradients of state variables as in CIT, but also on the time derivatives of fluxes, more explicitly,

$$
X_{i}=\nabla \cdot \frac{\partial s}{\partial \alpha}_{i}+m_{i j} \frac{\partial J_{j}}{\partial t}
$$

with

$$
J_{i}=\sum_{j} L_{i j} X_{j}
$$

By comparison, the term $\partial s / \partial \dot{\alpha}_{i}$ in the Onsager-Machlup approach (see expression $(5.5))$ is now replaced in $(5.15)$ by $\partial J_{i} / \partial t$ but $J_{i}$ is not given by $\dot{\alpha}_{i}$. Moreover, in both formalisms, the phenomenological equations are of a different nature which allows Garcia-Colin and Rodriguez to conclude that Gyarmati's formulation is not a consequence of the Onsager-Machlup theory [Garcia-Colin 1988a]. With regard to the EIT formulation by Lebon, Jou, and Casas-Vazquez, expressions (5.15) are viewed by Gyarmati as constitutive equations. Time evolution relations for the $J_{i}$ 's are not explicitly obtained nor sought. Moreover, Gyarmati keeps for the entropy flux the classical expression $\boldsymbol{q} / T$ and his constitutive equations do not contain terms in the gradients of the flux variables (as in expressions (4.16) to (4.19)) and are therefore less general. Finally, the symmetry of the phenomenological coefficients cannot be proved as the demonstration requires that the fluxes $J_{i}$ are the time derivatives of the $\alpha_{i}$ variables. Gyarmati's formalism was developed by his collaborators as Verhas who proposed a generalization of the entropy flux [Verhas 1983].

\section{Relativistic versions}

The question of a finite speed of propagation of thermal and viscous signals is especially compelling from the point of view of relativity theory. Therefore, it is logical that this topic received special attention. Early relativistic approaches to hydrodynamics and non-equilibrium thermodynamics have been proposed by Eckart and by Landau and Lifshitz [Eckart 1940; Landau 1958]. However, these theories suffer from two main drawbacks; first, they predict an infinite speed for the propagation of thermal and viscous signals, which is not compatible with the principle of causality and also with some experimental data, but it is certainly intolerable in any relativistic 
theory which imposes that no material body can move faster than the speed of light. Secondly, the transport equations of the first-order theory contain some undesirable generic instabilities: for example, small-amplitude disturbances from equilibrium diverge exponentially with time on very short time scales [Hiscock 1985]. These shortcomings are overcome by using relativistic versions of EIT, e.g. [Israel 1976; Jou 1993; Müller 1998].

In the relativistic context, the energy and mass balance equations can be formulated as

$$
T_{, \mu}^{\lambda \mu}=0, \quad N_{, \mu}^{\mu}=0
$$

with $T^{\lambda \mu}$ the energy-momentum tensor and $N^{\mu}$ the particle-flux vector. The indices run from 1 to 4 ( $\mu=1,2,3$ for space, $\mu=4$ for time) and a comma denotes a timespace derivative, in this section, summation convention on repeated indices will be used. The entropy density per unit volume $s$ and the entropy flux $\boldsymbol{J}^{s}$ form an entropy four-vector $s^{\mu}\left(s, \boldsymbol{J}^{s}\right)$, and the second law is expressed as the four divergence of $s^{\mu}$;

$$
s_{, \mu}^{\mu}>0
$$

In the framework of relativistic EIT, the entropy four-vector is given by

$$
s^{\mu}=s_{N} u_{N}^{\mu}+T^{-1} q_{N}^{\mu}-Q_{N}^{\mu}
$$

where $u_{N}^{\mu}$ is the unit time-like vector parallel to $N^{\mu}, s_{N}$ the local-equilibrium entropy in the particle frame, $q_{N}^{\mu}$ the heat flux vector, while $Q_{N}^{\mu}$ contains the contribution of the flux variables to the entropy and the entropy flux, namely (e.g. [Israel 1976, 1979])

$$
T Q_{N}^{\mu}=(1 / 2) u_{N}^{\mu}\left(\beta_{0} p^{v 2}+\beta_{1} q^{\alpha} q_{a}+\beta_{2} P^{v \lambda \beta} P_{\lambda \beta}^{v}\right)-a_{0} p^{v} q^{\mu}-a_{1} P_{\nu \mu}^{v} q^{\nu} .
$$

The first term of the right-hand side of (6.4) represents the second-order contributions of the fluxes to the entropy, whereas the second and third terms are the additional contributions to the entropy flux.

\subsection{Müller (1969, 1986): Relativistic extended thermodynamics}

Soon after the publication of his first papers on EIT, Müller formulated a preliminary relativistic version of them in a paper entitled "Towards relativistic thermodynamics" (1969). In this work, the consequences of a hyperbolic heat conduction equation were framed into their relativistic consistency. An application to simple conducting fluids was published in 1972 [Alts 1972]. Some years later, together with Liu and Ruggeri, Müller proposed a relativistic version of rational extended thermodynamics [Liu 1986]. The classical balance equations of mass, momentum, and energy are written in their relativistic form as well as the balance laws of the higher-order fluxes. The differences with the classical non-relativistic theory are that one is faced with 14 field equations instead of 13 in the non-relativistic case and that the formalism requires first and second order moments against distribution functions up to the fourth order in the non-relativistic scheme. Explicit field equations are derived with only four parameters left unknown: the thermal equation of state, the heat conductivity, the bulk and shear viscosities, which can be obtained either from statistical mechanics or from experimental data. Specific applications are to the non-degenerate relativistic gas, the ultra-relativistic gas and mixture of relativistic fluids [Hutter 1975; Müller 1998]. 


\subsection{Kranys $(1972,1977)$, Sieniutycz $(1984,1992)$ : Partial differential equations and variational formulations}

Kranys left Czechoslovakia in 1968 when Prague was occupied by the Warsaw Pact armies and moved to Montreal in Canada. He focused his attention on different subjects widely related to EIT, like hyperbolicity and its agreement with the causality principle and the relativity principle, requiring that one cannot exceed the speed of light in vacuo [Kranys 1972, 1977]. A general form of the hyperbolic operators for the most frequently used equations in mathematical physics was proposed. Hyperbolization of several equations as the Fourier, Stokes, Korteweg-deVries, Klein-Gordon, Schrödinger and Maxwell electromagnetic relations [Kranys 1989] were worked out. Kranys suggested also a Lagrangian formulation of the equations that he had studied. Describing dissipative processes by means of a Lagrangian formalism has been a subject of great interest during the seventies and eighties because there are many advantages in using them. For instance, they are the basis to the construction of variational principles. In that respect, let us also mention the contributions by Sieniutycz, from Warsaw Technological University and Berry from Chicago University [Sieniutycz 1984, 1992]. However these variational formulations have been the subject of severe criticisms [Finlayson 1972; Lebon 1980b] as they are not "true" variational principles in the sense that some quantities have to be kept fixed during the variational procedure.

\subsection{Israel (1976): Relativistic kinetic theory}

Relativistic thermodynamics was revisited in 1976 by Israel, then at the California Institute of Technology during a leave of absence from the University of Alberta in Canada. At this time, Israel was not aware of Müller's work. Israel was able to derive macroscopic transport equations akin to the evolution equations (4.17)-(4.19) of nonrelativistic EIT [Israel 1976]. He also discussed fluid mixtures. Restrictions imposed by the second law, expressed by (6.2), allowed him to derive explicit relations for the entropy flux and the entropy production. The results of Israel's phenomenological approach are shown to be parallel to the relativistic theory based on Boltzmann's equation. Since Israel's first article of 1976, a great number of papers was generated in relation with the relativistic kinetic theory and some astrophysical applications, as for instance supernova explosions, (e.g. [Stewart 1977; Israel 1979]. In particular, Israel's results were used in the analysis of the cosmological evolution of viscoelastic matter by Belinskii and collaborators [Belinskii 1979], opening the way to a fruitful field of applications of extended thermodynamics.

\subsection{Pavón, Jou, and Casas-Vázquez (1980): A relativistic version of Cattaneo's equation}

Pavón, Jou, and Casas-Vázquez at the Autonomous University of Barcelona, still unaware of Israel's work, proposed an extension of Cattaneo's equation within the relativistic context, by starting from the assumption that entropy and entropy flux are heat-flux dependent quantities [Pavon 1980]. The generalized Gibbs equation takes the form

$$
T D s=D u+p D v+a q^{\mu} D q_{\mu},
$$

where $D=u^{\nu} D_{\nu}$ is the relativistic invariant derivative and $a$ is a phenomenological coefficient. Combining the above result with the energy conservation law

$$
\rho D u+p D v+c^{-1}\left(d_{\mu} q^{\mu}+u^{\mu} D u_{\mu}\right)=0,
$$


where $d_{\mu}$ is the fourth-order space-time derivative operator, one obtains expressions for the entropy flux and the entropy production,

$$
J^{s \nu}=(1 / c T) q^{\nu}, \quad s_{\mu}^{\mu}=(1 / c T) q^{\mu}\left(d_{\mu} T+T D u_{\mu}-c \alpha T D q_{\mu}\right),
$$

suggesting the following relativistic form of the Cattaneo equation

$$
q^{\mu}=-\lambda \Delta^{\mu \nu}\left(d_{\nu} T+T D u_{\nu}-c \alpha T D q_{\nu}\right) .
$$

The phenomenological coefficient $\alpha$ is identified as $\alpha=-\tau / \lambda T$. Note the presence of the term in the acceleration $D u_{\nu}$, which is not present in the classical versions of Cattaneo's law. A particular attention has been devoted to viscous cosmological models and their effects on the dynamics of the universe. Formulations of an extended Gibbs relation for nuclear matter and relaxation transport equations have proven to be of interest in the hydrodynamic analysis of collisions between heavy ions: the analysis of these collisions is helpful to determine the nuclear equations of state and to study the phase transition from the hadronic matter to the quark-gluon plasma [Griffin 1976; Stocker 1986; Koide 2007]. It was observed that generally the duration of the collisions between heavy nuclei is only one order of magnitude higher than the mean free time of nucleon collisions inside the nuclei which indicates that relaxation effects, as described by EIT, are important. Furthermore, during collisions the nuclei are far from equilibrium, so that non-equilibrium corrections of the equations of state may considerably affect the energy required for the phase transition in nuclear matter.

\section{Kinetic and statistical mechanics approaches}

We have already emphasized the role of kinetic theory as a source of motivation and inspiration for generalized transport equations. Furthermore, kinetic theory provides a version of the second law through the well-known $H$-theorem. The relation between the quantity $H$ and the thermodynamic entropy is clear in equilibrium states, but this is no longer true far from equilibrium. The microscopic "entropy", or $H$ function, is defined by (3.12). Out of equilibrium, let the distribution function be given by

$$
f=f e q(1+\Phi)
$$

with $\Phi$ a "first-order" non-equilibrium contribution, depending for instance on the heat and momentum fluxes. Introducing (7.1) in expression (3.12) of the entropy leads, up to and including second-order terms in $\Phi$, to

$$
\rho s=\rho s_{e q}-\left(k_{B} / 2\right) \int f_{e q} \Phi^{2} d c .
$$

The merit of the above result is to shed some light on the range of validity of the local equilibrium hypothesis. Indeed, it is well known that the classical transport equations depend only on the first-order correction in $\Phi$ but, as seen in (7.2), such a first-order correction is absent from expression (7.2) whose first correction is of second order in $\Phi^{2}$. Thus, unless one is interested in a generalization of the transport equations or in second or higher order terms in the entropy, the local-equilibrium entropy remains satisfactory. It is also worth to stress that the rate of production of the truncated entropy (7.2) is positive for linearized transport laws, but not in general for nonlinear evolution equations, in contrast to the general entropy production resulting from the use of the general definition (3.12).

Classical kinetic theory is useful in obtaining expressions for the transport coefficients for a diversity of physical systems, or, in more sophisticated approaches, to 
formulate generalized transport equations with higher-order terms; however, in such developments, no special attention is paid neither to the definition of temperature, which is simply defined in terms of the kinetic theory of the particles, nor to the entropy. At the beginning of the 1980's, these questions received special attention as illustrated by the contributions to the kinetic foundations of extended thermodynamics by García-Colin and Eu as discussed in Sections 7.1 and 7.2.

\subsection{The Mexican school (Garcia-Colin)}

Garcia-Colin was one of the most prominent scientists in Mexico during the last 50 years. After doctoral studies at the University of Maryland, he was a member of the Colegio Nacional, the equivalent of the National Academy of Sciences and occupied several positions at the Universidad Nacional Autonoma de Mexico (UNAM) and the Universisdad Autonoma Metropolitana (UAM). He passed away in October 2012. A critical discussion of the main ideas behind EIT based on kinetic theory and statistical mechanics can be found in some papers by Garcia-Colin and his collaborators (e.g. [Garcia-Colin 1984, 1988b, 1995]).

The first criticism of Garcia-Colin against EIT is about the fact that the fluxes express the response of the system to the action of a force (like the gradient of temperature) rather than the measure of intrinsic properties. Based on Grad's development, it was suggested to use the higher moments $a_{i}$ of the distribution function which are in general a combination of the physical fluxes rather than the fluxes themselves. A different choice, meeting Garcia-Colin's objection, was recently proposed; instead of the heat flux variable $\boldsymbol{q}$ in the problem of heat transport, a renormalized flux variable $\boldsymbol{q}_{n}$ was preferred taking into account the constitutive properties of the material like relaxation time and heat conductivity [Lebon 2008a]. A second controversial feature was about the identification of the $s$-function with an entropy function. There exist, however, strong arguments from the kinetic and information theories (e.g. [Jou 1993]) indicating that $s$ may indeed be identified with a non-equilibrium entropy; further justifications were brought forward by $\mathrm{Eu}$, using the notion of uncompensated heat proposed by Clausius [Eu 1992]. But more fundamentally, Garcia-Colin asked whether the property that $\sigma^{s}$ is positive definite is generally valid. He argued that the inequality $\sigma^{s} \geqslant 0$ has no meaning beyond the local equilibrium hypothesis. This is, of course, a crucial point as it concerns not only EIT, but also other nonequilibrium approaches as Rational Thermodynamics or internal variable theories. It is our opinion that the positiveness of the entropy production must be considered as a principle to be accepted, unless contradicted by experiments. Despite these controversial features, Garcia-Colin spent much effort to lay foundations of EIT on the general principles of the kinetic theory and statistical mechanics. It was determined by Garcia-Colin and Lopez de Haro to which extent the constitutive relations of EIT cope with the Green-Kubo relations of statistical Mechanics and the Chapman-Enskog scheme [Garcia-Colin 1982], more particularly with the Burnett and super Burnett regimes [Garcia-Colin 1984].

\subsection{Eu's modified moment method (1980)}

This method rests on Grad's original approach and was introduced by Eu in 1980 . This author spent the essential period of his scientific activity at McGill University in Montreal before becoming a consultant of the Scientific Policy Department of SouthKorea, his native country. Eu's approach has undergone several versions with the 
latest version published in an extensive monograph on the subject [Eu 1992]. The variables are the fluxes and the tensorial higher moments $M^{i}$ defined by

$$
M^{i}=\left\langle m c^{i} f(\boldsymbol{r}, t, \boldsymbol{c})\right\rangle,
$$

with \langle\rangle$=(1 / n) \int f d c$ and $c^{i}$ standing for the dyadic product $c c$, the third-order vectot $(\boldsymbol{c . c}) \boldsymbol{c}$, etc. Now, instead of Grad's expansion in terms of Hermite polynomials, $\mathrm{Eu}$ proposes for $f$ the so-called canonical form

$$
f=C \exp \left[-\beta\left(H_{0}+H_{1}\right)\right]
$$

where $H_{0}=(1 / 2) m c^{2}+V(r), V(r)$ being a conservative potential energy and

$$
H_{1}=\sum_{\alpha=1}^{r} X^{(\alpha)} \otimes h^{(\alpha)},
$$

the $h^{(\alpha)}$ 's are essentially the Hermite polynomials of Grad's method and the $X^{(\alpha)}$ some thermodynamic forces which, at the lowest order are given by $\boldsymbol{X}^{(1)}=-\left(\tau_{1} / 2 k_{B} \lambda T^{2}\right) \boldsymbol{q}$, $\left.\boldsymbol{X}^{(2)}=-\tau_{2} / 4 k_{B} \eta T\right) \stackrel{0}{\boldsymbol{P}^{v}}$, symbol $\otimes$ denotes contraction or inner products. Grad's approach may be recovered after linearization of the exponential expression (7.4) around the local equilibrium distribution given by $\exp \left(-\beta H_{0}\right)$. After rather lengthy but straightforward calculations, one obtains for the entropy production a bilinear form

$$
\sigma^{s}=\frac{1}{T} \sum_{\alpha} X^{(\alpha)} \otimes \Lambda^{(\alpha)},
$$

with $\Lambda^{(\alpha)}=\left\langle h^{(\alpha)} J(f f)\right\rangle$ and $J(f f)$ standing for the collision term. The entropy flux is not given by the classical expression $\boldsymbol{q} / T$ but by the more intricate relation

$$
\boldsymbol{J}^{s}=\frac{\boldsymbol{q}}{T} \sum_{\alpha} X^{(\alpha)} \otimes \phi^{(\alpha)},
$$

with $\phi^{(\alpha)}=\left\langle\boldsymbol{c} h^{(\alpha)} f\right\rangle$. It is interesting to note that Gibbs equation has the same structure as in EIT, namely

$$
T d s=d u+p d v+\sum_{\alpha} X^{(\alpha)} \otimes d \Phi^{(\alpha)}
$$

where $\Phi^{(\alpha)}=\left\langle h^{(\alpha)} f\right\rangle$. The gist of the ideas underlying Eu's approach is the same as in the more classical approaches by Jou, Lebon, and Müller's groups, i.e. enlargement of the space of state variables, restrictions placed by the second law, deep connection with kinetic theory. The merit of Eu's theory is its ability to cover the non-linear domain and, in particular, to yield constitutive relations predicting finite saturation of the fluxes within the limit of high values of the forces. This saturation is consistent with finite propagation of signals, and could be imposed as an additional requirement in the phenomenological approach of EIT. Eu was able to apply his theory not only to monatomic ideal gases but also to dense fluids, polymer solutions and other complex systems. The main difficulties with Eu's formalism is to assign an unequivocal physical interpretation of the several undetermined parameters and to propose a way of measuring them. Moreover, the evolution equations of the $X^{(\alpha)}$ are rather complicated and have been solved only in rather simple cases. Nevertheless, at the simplest linear approximation, all the methods examined previously are converging in the sense that all of them lead to Cattaneo-Vernotte-Maxwell's type equations. 


\subsection{The MAXENT-NSOM approach (Luzzi and Vasconcellos)}

Interesting justifications of the statistical foundations of EIT are also provided by a statistical-mechanics approach, the so-called MAXENT-NSOM (Maximum EntropyNonequilibrium Statistical Operator Method) proposed by Luzzi and his collaborators at the university of Campinas (Brazil) [Luzzi 2001, 2002]. This model rests on a variational principle, namely the Jaynes (1963) maximization of the information statistical entropy. The requirement that entropy should be maximized in non-equilibrium steady states is not contradictory with maximization in equilibrium states, because it imposes restrictions not only on the internal energy and the number of particles (as in the macrocanonical distribution function) but also on fluxes present in the system (for instance, energy flux, matter flux, and momentum flux). The maximum value of the entropy under these additional requirements on the fluxes is less than the value of the entropy in equilibrium states. Thus, equilibrium states truly maximize the total entropy, under vanishing values of the fluxes. The higher the number of the non-vanishing fluxes, the lower is the corresponding maximum entropy. Maximum entropy theories introduce Lagrange multipliers conjugated to the several constraints acting on the system. The corresponding Lagrange multipliers are equivalent to those found in Müller and Liu's work, although motivated by different arguments. The Lagrange multipliers conjugate to the classical variables have a physical meaning, unlike the Lagrange multipliers conjugate to the fluxes which cannot generally be given a straightforward interpretation. They may, however, be interpreted by comparison with the generalized Gibbs equation of EIT. The expressions obtained from the generalized flux-dependent entropy are not limited to second-order terms, as in the simplest version of phenomenological EIT, but are strongly non-linear and non-local.

\section{Other faces of non-equilibrium thermodynamics}

\subsection{Rational thermodynamics}

As EIT was knowing its first steps in the early seventies, non-equilibrium thermodynamics was essentially dominated by CIT for one part and by rational thermodynamics for the other part. The roots of this theory are found in the developments of rational mechanics. Axiomatic aspects with theorems, axioms, and lemmas are dominating the account. The foundational papers are these of Coleman and Noll and Coleman published in 1963 and 1964 respectively [Coleman 1963, 1964], the name "Rational Thermodynamics" was dubbed by Truesdell a few years later [Truesdell 1966, 1969]. Although the formalism is mostly concerned with deformable solids with memory, other classes of systems, including fluids, chemical reactions, and shock waves, have also been investigated. The main objective is to put restrictions on the form of the constitutive equations by application of formal statements of thermodynamics. The founders of rational thermodynamics consider it as an autonomous branch arguing that a justification of the foundations and results must ultimately come from the theory itself. Rational thermodynamics was appreciated by the community of pure and applied mathematicians who were attracted by its axiomatic vision of continuum mechanics. The popularity of the theory failed at the end of the eighties because it was disregarded by physicists for its lack of physical background and by engineers because the constitutive equations take the form of functionals, which are not very tractable in practical situations (see the critical discussions by Lavenda and Woods [Lavenda 1979; Woods 1981]). It appears also that the signs of some rheological coefficients as in the Rivlin-Ericksen model are contradicted by experience. Moreover, rational thermodynamics predicts instabilities for non-Newtonian fluids as it leads to an exponential growth of shear strain rate for a vanishing stress tensor [Müller 2012]. 


\subsection{Internal variables theory}

An alternative formalism was given by the internal variable theory (IVT), sometimes named "hidden variable theory" (e.g. [Coleman 1967; Mandel 1978; Kestin 1980; Bampi 1984; Maugin 1994]). The basic idea is to complement the state of variables of classical non-equilibrium thermodynamics by extra variables describing the internal motions or (and) the internal microstructure. The foundations of IVT can be traced back to the kinetic theory with internal degrees of freedom (e.g. [Kirkwood 1967]) which emerged on the kinetic theory of polymeric fluids by Hand and Bird among others [Hand 1962; Bird 1987]. To relate the theory of Kirkwood to thermodynamics was not a trivial task as it implies that one cannot express the entropy in terms of a polynomial in the fluxes. First attempts [Lhuillier 1979, Grmela 1987] were made by Lhuillier and later by Grmela and Lye who suggested to formulate the corresponding entropy in the form $s=(1 / 2) \ln \operatorname{det} c$ where $\boldsymbol{c}=\int \boldsymbol{R} \boldsymbol{R} f d \boldsymbol{R}$ is the conformation or Hand's tensor, and $\boldsymbol{R}$ the end-to-end macromolecular distance between the molecules of the polymer chains. IVT has proven to be well adapted to the description of chemical reactions, plastic bodies, polymer solutions and electromagnetic bodies.

In contrast to EIT where the variables are well identified from the start, the main characteristic of internal variables is that usually there is a priori no reference to their physical nature, it is only at the end of the procedure that, in general, their physical meaning emerges. In contrast to external variables, the internal variables are not coupled to external forces, they do not contribute to the mechanical work and are consequently not controllable. Although not controllable, internal variables are nevertheless measurable by the effects produced inside the system: as an example, in plasticity, the internal variable is linked to the motion of dislocations and can be measured but not controllable.

A particular case is to consider the fluxes themselves as internal variables, leading to evolution equations for the fluxes, as in EIT. Let us illustrate this situation with two examples. The first one refers to polymer solutions, where it is logical to take the molecular configuration as an internal variable. This is usually achieved by means of the conformation tensor. In several macroscopic problems, where the tangential pressure on the boundaries of the system is controlled, the viscous pressure tensor of EIT appears as a more natural variable and has been preferred by some researchers [Jou 2000]. The second example is heat transport in superfluid helium II, where the heat flow may be considered either as an internal variable by itself, or may be related to the relative velocity between the normal and the superfluid components selected as internal variable [Mongiovi 1991, 1992; Jou 2000]. In practical problems, one observes a strong similarity between the final results whatever the choice of the variables (the viscous pressure or the heat flux in the case of EIT, the conformation tensor, or the relative velocity of the normal and superfluid components in the internal variables theory). This shows that EIT may be compared in a natural way with other non-equilibrium theories, especially at the microscopic level, beyond the classical description provided by the Boltzmann equation for ideal gases with predominant binary collisions.

\subsection{Hamiltonian structures}

Hamiltonian structures have been identified at various levels of description: the microscopic one (classical mechanics), the kinetic theory, and some macroscopic approaches (as, for instance, hydrostatics or equilibrium thermodynamics). It is natural to ask whether such a structure is preserved in more sophisticated descriptions and, 
more particularly, in non-equilibrium thermodynamics. An early Hamiltonian version of non-equilibrium thermodynamics was proposed by Grmela in a "Joint Summer Conference on the Mathematical Sciences on Fluids and Plasmas: Geometry and Dynamics" held in Boulder in 1983 [Grmela 1984]. The subject can be traced back to works by Clebsch and continued by Arnold [Clebsch 1859; Arnold 1966]. The first papers were based on one single generator, and the time evolution of the basic variables was expressed in terms of a non-dissipative Poisson bracket plus a dissipative bracket (e.g. [Beris 1994]). These formulations were more recently superseded in some aspects by what is now known as GENERIC (General Equation for the Non-Equilibrium Reversible-Irreversible Coupling), whose latest developments can be found in Öttinger's book [Öttinger 2005] and in papers by Grmela [Grmela 2010, 2014]. One of the main merits of a Hamiltonian structure is that it provides restrictions on the reversible dynamics while the other thermodynamic theories, among which CIT, RT and EIT, place only restrictions on the irreversible parts of the constitutive equations. It is also worth stressing that the evolution equations of the fluxes of EIT (at least in the case of an incompressible fluid) share the property of possessing a Hamiltonian structure, as shown by Grmela and Lebon [Grmela 1990].

\section{The Bellaterra meeting (1983): The first meeting devoted to EIT}

Several "Schools" were organized in the city of Bellaterra, the seat of the Universitat Autonoma Barcelona, starting in 1983 with the Escuela de Thermodinamica de Bellaterra, whose proceedings were published in [Casas-Vazquez 1984].

The objective of these organizations was twofold: first, to couple the pedagogical trends of a school with the incentive of fresh information on topics of actual interest in non-equilibrium thermodynamics; second, to offer an overview of the several hundred papers that had been published by the diverse groups.

At this first international congress organized in 1983, particular emphasis was put on the early developments of EIT. The multi-faced aspects of the foundations were presented by Nettleton, Müller, the Liège-Barcelona school (Lebon, Casas-Vazquez, Jou), Lengyel (Gyarmati's school), Garcia-Colin and Eu. An important part of the Conference was concerned with selected applications and with a comparison with other non-equilibrium approaches such as rational thermodynamics and the internal variable theory. Relations with the fluctuation theory and the kinetic theory of gases were also the subject of various contributions. The possibility of having a direct contact and live discussion amongst the several promoters of extended thermodynamics fostered its future presentations as a full-fledged theory, motivated by microscopic foundations and rich in applications. These presentations came out a few years later, in the form of reviews [Jou 1988; Nettleton 1995] and books [Eu 1992; Jou 1993, 1996, 2001, 2010; Lebon 2008b; Müller 1993, 1998; Sieniutycz 1994]. The 1983 school was paralleled and followed by other international conferences with the objective to open the concepts and applications of EIT to a wider audience.

After the 1983 Conference, two main trends of thought have emerged; the socalled Extended Rational Thermodynamics (ERT) headed by Müller and Ruggeri and the Extended Irreversible Thermodynamics formalism (EIT) as developed by the Liège-Barcelona school. EIT generalizes the Gibbs equation of CIT and does not require balance equations a priori, while ERT assumes a set of evolution equations based on the kinetic theory. EIT is more general but ERT is appropriate for studying monatomic, polyatomic and dense gases and shock waves. The last versions are summarized in the monographs co-authored by Müller and Ruggeri [Müller 1998] and Jou [Jou 1993, 2010] respectively with the aim to consolidate the theory as a broad and well-founded framework, with many related facets. 


\section{Concluding remarks}

Through its several faces outlined in Section 5, Extended Thermodynamics has the advantage of proposing a formalism that is accessible to a wide community of researchers and applicants of physics, chemistry, biology, mathematics and engineering science. It is a well-structured mesoscopic formalism, whose foundations have been confirmed by kinetic theory, non-equilibrium statistical mechanics, and fluctuation physics. The parallelism between Extended Thermodynamics and information theory should be emphasized as both approaches use a generalized entropy, which is not necessarily limited to second order corrections. Extended Thermodynamics also elucidates the physical meaning of the Lagrange multipliers introduced to take into account the non-equilibrium constraints. As it goes beyond the local-equilibrium hypothesis, Extended Thermodynamics allows us to describe a wide variety of phenomena and processes, not (or only partially) covered by other thermodynamic formalisms.

Extended Thermodynamics is not confined to a limited number of places and schools, such as Barcelona, Berlin, Bologna, Liège, but it has spread over a much enlarged area, including Budapest (Gyarmati, Van, and Verhas), Catania, Messina, and Palermo, (contributions by Anile, Valenti, Palumbo, Barbera, Restuccia and Mongiovì), Köln (Galenko), Montreal (Eu and Grmela), Campinas (Luzzi and Vasconcellos), Warsaw (Sieniutycz), attesting for the universality of the problems dealt with in this theory.

The applications discussed in the present review represent only a part of a vast and varied domain of applicability with emphasis on

- memory effects (fast processes, polymers, superfluids);

- non-local effects (micro- and nano-materials);

- non-linear effects (high powers, shock waves).

Actually, much efforts are paid to a discussion of the mathematical foundations [Cimmelli 2014], to rarefied gases [Struchtrup 2005] and the formulation of boundary conditions for high order moments [Müller 2003; Barbera 2004], heat transport at micro- and nano-scales is also the subject of intense activity (e.g. [Alvarez 2012; Lebon 2014]).

We have seen that Extended Thermodynamics is by no way the unique theory of non-equilibrium thermodynamics and that the diversity of schools in non-equilibrium thermodynamics is considerably rich (e.g. [Muschik 2007]). In addition to the classical formulation of irreversible processes as formulated by Onsager, de Groot, Mazur, and the Brussels school, we have briefly discussed the basic ideas of Rational Thermodynamics, mainly advocated by Coleman, Noll, and Truesdell, the GENERIC formalism, originally developed by Grmela and Öttinger as well as the hidden variable theory, worked out by Kestin, Maugin, Lhuillier, Bampi and Morro, among others. Answering the question "what is the best approach?" is highly subjective; it depends not only on the nature of the system to be analyzed but also on the tools available to the researcher, in addition to his goals, scientific education, and environment. The discussion is far from being closed. Up to now, most emphasis has been put on fluids while thermodynamics of solids has been the subject of less attention and asks for more developments. Several outstanding fundamental questions such as that of the uniqueness of non-equilibrium entropy and temperature, the status of the second law of thermodynamics, or the selection of the relevant state variables, to mention only the most important problems so far were only partly addressed and call for more definitive solutions.

Acknowledgements. The authors wish to thank warmly Prof. Jose Casas-Vazquez (UAB Barcelona), our collaborator for more than 40 years, for his interest in this work and for his 
still relevant observations. We also are indebted to our colleagues T. Sauer and K. Hutter and two anonymous referees whose observations and recommendations have contributed to improve the manuscript. D.J. acknowledges the financial support of the Spanish Ministry of Economy and Competitiveness under grant FIS2012- 33099 and of the Direcció General de Recerca of the Government of Catalonia under grant 2009 SGR 00164.

\section{References}

Alts, T. and I. Müller. 1972. Relativistic thermodynamics of simple heat conducting fluids. Arch. Rat. Mech. Anal. 48: 245-273.

Alvarez, F.X., A.V. Cimmelli, D. Jou and A. Sellitto. 2012. A mesoscopic description of boundary effects in nanoscale heat transport. Nanoscale Syst. MMTA 1: 112-142.

Anile, A.M. and S. Pluchino. 1984. Linear waves modes for dissipative fluids with rate type constitutive equations. Meccanica 19: 204-110.

Arnold, V. 1966. Sur la géometrie différentielle des groupes de Lie de dimension infinie et ses applications à l'hydrodynamique des fluides parfaits. Ann. Inst. Fourier 16: 319-361.

Bampi, F. and A. Morro. 1984. Non equilibrium thermodynamics: a hidden variable theory. In: Lecture Notes in Physics, Vol. 199. Springer, Berlin.

Barbera, E., I. Müller, D. Reitebuch and N.R. Zhao. 2004. Determination of boundary conditions in extended thermodynamics via fluctuation theory. Continuum Mech. Thermodyn. 16: 411-425.

Belinskii, V.A., S. Nikomarov and I.S Khalatnikov. 1979. Investigation of the cosmological evolution of a viscoelastic matter with causal thermodynamics. Sov. Phys. JETP 50: 213-221.

Belousov B.P. 1958. A periodic reaction and its mechanism. Shornik Referatov po Radiacionnoii Medicine za. (Collection of Abstracts on Radiation Medicine), pp. 145147. Moscow.

Bénard, H. 1900. Les tourbillons cellulaires dans une nappe liquide transportant la chaleur par conduction en régime permanent. Rev. Gén. Sci. Pures et Appliquées 11: 1261-1271.

Beris, A.N. and B.J. Edwards. 1994. Thermodynamics of Flowing Systems with Internal Microstructures. Oxford Sci. Pub., Oxford.

Bird, R.B., C.F. Curtiss, R.C. Armstrong and D. Hassager. 1987. Dynamics of Polymer Liquids, $2^{\text {nd }}$ edition, Vol. 2. Wiley, New York.

Boltzmann, L. 1872. Weiteren Studien über das Wärmegleichgewicht zwischen Gasmolekülen. Sitzungsberichte der Akad. der Wissensch. Wien Abt. II: 275-370.

Boltzmann, L. 1895, 1898. Vorlesungen über Gastheorie I und II. Verlag Metzger und Wittig, Leipzig.

Bubnov, V.A. 1976. Wave concepts in the theory of heat. Int. J. Heat Mass Transfer 19: $175-184$.

Carnot, S. 1824. Réflexions sur la puissance motrice du feu et sur des machines propres à développer cette puissance. Librairie Bachelier, Paris.

Casas-Vazquez, J., D. Jou and G. Lebon. (eds.). 1984. Recent Developments in NonEquilibrium Thermodynamics. In: Lecture Notes in Physics, Vol. 199. Springer, Berlin.

Casas-Vazquez, J. and D. Jou. 2003. Temperature in nonequilibrium states: a review of open problems and current proposals. Rep. Prog. Phys. 66: 1937-2023.

Casimir, H.B.G. 1945. On Onsager's principle of microscopic irreversibility. Rev. Mod. Phys. 17: 343-350.

Cattaneo, C. 1948. Sulla conduzione del calore. Atti Seminario Mat. Fis. Univ. Modena 3: 83-101.

Chapman, S. and T.G. Cowling. 1970. The Mathematical Theory of Non-uniform Gases. Cambridge Univ. Press, Cambridge.

Chester, M. 1963. Second sound in solids. Phys. Rev. 131: 2013-2015.

Chester, M. 1966. High-frequency thermometry. Phys. Rev. 145: 76-80.

Cimmelli, A., D. Jou, T. Ruggeri and P. Van. 2014. Entropy principle and recent results in non-equilibrium theories. Entropy 16: 1756-1807. 
Clausius, R. 1854. Über eine veränderte Form des zweiten Hauptsatzes der mechanischen Wärmetheorie. Poggendorff's Annalen der Physik 93: 481-506.

Clausius, R. 1865. Über verschiedene für die Anwendung bequeme Formen der Hauptgleichungen der mechanischen Wärmetheorie. Poggendorf's Annalen der Physik 125: $353-400$.

Clebsch, A. 1859. Über die Integration der hydrodynamischen Gleichungen. J. Reine Angew. Math. 56: 1-10.

Coleman, B.D. and C. Truesdell. 1960. On the reciprocal relations of Onsager. J. Chem. Phys. 33: 28-31.

Coleman, B.D. and W. Noll. 1963. The thermodynamics of elastic materials with heat conduction and viscosity. Arch. Rat. Mech. Anal. 13: 167-178.

Coleman, B.D. 1964. Thermodynamics of materials with memory. Arch. Rat. Mech. Anal. 17: $1-46$.

Coleman, B.D. and M.E. Gurtin. 1967. Thermodynamics with internal state variables. $J$. Chem. Phys. 47: 597-613.

Courant, R. and D. Hilbert. 1962. Methods of Mathematical Physics. J. Wiley, New York.

de Groot, S.R. 1951. Thermodynamics of Irreversible Processes. North-Holland, Amsterdam.

de Groot, S.R. and P. Mazur. 1962. Non-equilibrium Thermodynamics. North-Holland, Amsterdam.

Denbigh, R.G. 1950. The Thermodynamics of the Steady state. Wiley, New York.

Dufour, L. 1873. Über die Diffusion der Gase durch poröse Wände und die sie begleitenden Termperaturveränderungen. Ann. der Phys. 148: 490-492 (translated from the original article published in French in Arch. Sci. Phys. Nat. Genève 45: 9-11).

Eckart, C. 1940. The thermodynamics of irreversible processes. Phys. Rev. 58: 267-269 and 58: $269-275$.

Eu, B.C. 1980. A modified moment method and irreversible thermodynamics. J. Chem.Phys. 73: $2958-2969$.

Eu, B.C. 1992. Kinetic Theory and Irreversible Thermodynamics. Wiley, New York.

Fick, A. 1855. Über Diffusion. Ann. Phys. 94: 59-86.

Finlayson, B. 1972. The Method of Weighted Residuals and Variational Principles. Acad. Press, New York.

Fourier, J.B. 1822. Théorie Analytique de la Chaleur. F. Didot, Paris.

Garcia-Colin, L.S. and M. Lopez de Haro. 1982. The Burnett equations in extended irreversible thermodynamics. J. Non-Equilib. Thermodyn. 7: 95-104.

Garcia-Colin, L.S., R.F. Rodriguez, M. Lopez de Haro, D. Jou and J. Casas-Vazquez. 1984. On the foundations of extended irreversible thermodynamics. J. Stat. Phys. 17: 465-484.

Garcia-Colin, L.S. and R.F. Rodriguez. 1988a. On the relationship between extended thermodynamics and the wave approach in thermodynamics. J. Non-Equilib. Thermodyn. 13: $81-94$.

Garcia-Colin, L.S. 1988b. Extended non-equilibrium thermodynamics, scope and limitations. Rev. Mexicana Fisica 34: 344-366.

Garcia-Colin, L.S. 1995. Extended irreversible thermodynamics: an unfinished task. Mol. Phys. 86: 697-706.

Gaspard, P. 1998. Chaos, Scattering and Statistical Mechanics. Cambridge Univ. Press, Cambridge.

Gibbs, W. 1875, 1878. On the equilibrium of heterogeneous substances. Transactions of the Connecticut Academy, pp. 108-248 and pp. 342-524.

Glansdorff, P. and I. Prigogine. 1964. On a general evolution criterion in macroscopic physics. Physica. 30: 351-374.

Glansdorff, P. and I. Prigogine. 1971. Thermodynamics of Structures, Stability and Fluctuations. Wiley, New York.

Grad, H. 1949. On the kinetic theory of rarefied gases. Commun. Pure Appl. Math. 2: 331407.

Grad, H. 1958. Principles of the kinetic theory of gases. In: Flugge S. (ed.) Hd. der Physik, Vol. XII. Springer, Berlin. 
Griffin, J.J. and K.K. Kan. 1976. Colliding heavy ions: nuclei as dynamical fluids. Rev. Mod. Phys. 48: 467-477.

Grmela, M. and C. Lye. 1987. Shear flow induced structural changes in polymeric liquid crystals. Phys. Lett. A 120: 282-285.

Grmela, M. 1984. Particle and bracket formulation of kinetic equations. Contemp. Math. AMS 28: 125-132.

Grmela, M. and G. Lebon 1990. Hamiltonian extended thermodynamics. J. Phys. A23: $3341-3351$.

Grmela, M. 2010. Multiscale equilibrium and nonequilibrium thermodynamics in chemical engineering. Adv. Chem. Eng. 36: 75-128.

Grmela, M. 2014. Contact geometry of mesoscopic thermodynamics and dynamics. Entropy 16: $1652-1686$.

Gurtin, M.E. and A.C Pipkin. 1968. A general theory of heat conduction with finite wave speed. Arch. Rational Mech. Anal. 31: 116-126.

Guyer, R.A. and J.A. Krumhansl. 1966. Solution of the linearized Boltzmann phonon equation. Phys. Rev. 148: 766-778 and 148: 778-788.

Gyarmati, I. 1970. Non-equilibrium Thermodynamics. Springer, Berlin.

Gyarmati, I. 1977. On the wave approach of thermodynamics and some problems of nonlinear theories. J. Non-Equilib. Thermodyn. 2: 233-260.

Haase, R. 1969. Thermodynamics of Irreversible Processes. Addison-Wesley, Reading MA.

Hand, G.L. 1962. A theory of anisotropic fluids. J. Fluid. Mech. 13: 33-46.

Hess, S. 1977. On nonlocal constitutive relations, continued fraction expansion for the wave vector dependent diffusion coefficient. Z. Naturforsch. 32a: 678-684.

Hiscock, W.A. and L. Lindblom. 1985. Generic instabilities in first-order dissipative relativistic fluids theories. Phys. Rev. D 31: 725-733.

Hutter, K. 1977. The foundations of thermodynamics, its basic postulate and implications. A review of modern thermodynamics. Acta Mechanica 27: 1-54.

Hutter, K. and I. Müller. 1975. On mixture of relativistic fluids. Helvetica Physica Acta 48: $1-24$.

Israel, W. 1976. Non stationary irreversible thermodynamics: a causal relativistic theory. Ann. Phys. (New York) 100: 310-331.

Israel, W. and J.M. Stewart. 1979. Transient relativistic thermodynamics and kinetic theory. Ann. Phys. (NY) 118: 341-372.

Jaynes, E.T. 1963. Information theory and statistical mechanics. In: Statistical Physics (Ford, W.K. ed.). Benjamin, New York.

Jou, D., J. Casas-Vazquez and G. Lebon. 1979. A dynamical interpretation of second-order constitutive equations of hydrodynamics. J. Non-Equilib. Thermodyn. 4: 349-362.

Jou, D., J.E. Llebot and J. Casas-Vazquez. 1982. Thermodynamic aspects of non- equilibrium fluctuations. Phys. Rev. A 25: 3277-3281.

Jou, D. 1983. Equacions de Gibbs generalitzades i extensió de la termodinamica de processos irreversibles. Institut d'Estudis Catalans, Barcelona (in Catalan language).

Jou, D., J. Casas-Vazquez and G. Lebon. 1993. Extended Irreversible Thermodynamics. First edition. Second edition 1996. Third edition 2001. Fourth edition 2010. Springer, Berlin.

Jou, D., J. Casas-Vazquez and M. Criado-Sancho. 2000. Thermodynamics of Fluids under Flow. Second edition 2011. Springer, Berlin.

Jou, D., G. Lebon and M. Criado-Sancho. 2010. Variational principles in thermal transport in nanosystems with heat slip flow. Phys. Rev. E 82: 031128.

Jou, D., J. Casas-Vazquez, G. Lebon and M. Grmela. 2005. A phenomenological scaling approach for heat transport in nanosystems. Appl. Math. Lett. 18: 963-967.

Joule, J.P. 1841. On the heat evolved by metallic conductors of electricity, and in the cells of a battery during electrolysis. Phil. Mag. 19: 275.

Kestin, J. and J. Bataille. 1980. Thermodynamics of solids. In: Continuum Models of Discrete Systems. University of Waterloo Press, Waterloo.

Kirkwood, J.C. 1967. Selected Topics in Statistical Mechanics. Gordon and Breach, New York. 
Koide, T., G.S. Denicol, P. Mota and T. Kodama. 2007. Relatisvistic dissipative hydrodynamics: a minimal causal theory. Phys. Rev. C 75: 034909.

Kranys, M. 1972. Kinetic derivation of non-stationary general relativistic thermodynamics. Nuovo Cimento B 8: 417-441.

Kranys, M. 1977. Hyperbolic elasticity of dissipative media and its wave propagation modes. J. Phys. A: Math.Gen. 10: 689-709.

Kranys, M. 1989. Casual theories of evolution and wave propagation in mathematical physics. Appl. Mech. Rev. 42: 305-322.

Lambermont, J. and G. Lebon. 1973. On a generalization of the Gibbs equation for heat conduction. Phys. Lett. A 42: 499-500.

Landau, L.D. and E.M. Lifshitz. 1958. Mechanics of Fluids. Addison Wesley, Reading, Mass.

Landau, L.D. and E.M. Lifshitz. 1980. Statistical Physics, 3rd edition, Pergamon, Oxford.

Lavenda, B. 1979. Thermodynamics of Irreversible Processes. McMillan, London.

Lebon, G. and J. Lambermont. 1973. Generalization of Hamilton's principle to continuous dissipative systems. J. Chem. Phys. 5: 2929-2936.

Lebon, G. 1978. Derivation of generalized Fourier and Stokes-Newton equations based on the thermodynamics of irreversible processes. Bull. Acad. Roy. Belgique 64: 456-472.

Lebon, G., D. Jou and J. Casas-Vazqez. 1980a. An extension of the local equilibrium hypothesis. J. Phys. A: Math. Gen. 13: 275-290.

Lebon, G. 1980b. Variational Principles in Thermomechanics. In: Recent Developments in Thermomechanics of Solids (Lebon, G. and Perzyna, P. eds.). CISM Courses and Lectures, Vol. 282, pp. 221-415. Springer, Wien, New York.

Lebon, G. and A. Cloot. 1989. Propagation of ultrasonic sound waves in dissipative dilute gases and extended irreversible thermodynamics. Wave Motion 11: 23-32.

Lebon, G and P.C. Dauby. 1990. Heat transport in dielectric crystals at low temperature; A variational formulation based on extended irreversible thermodynamics. Phys. Rev. A 42: $4710-4715$.

Lebon, G., M. Ruggieri and A. Valenti. 2008a. Extended thermodynamics revisited: renormalized flux variables and second sound in rigid solids. J. Phys. C 20: 025223.

Lebon, G., D. Jou and J. Casas-Vazquez. 2008b. Understanding Non-equilibrium Thermodynamics, Foundations, Applications, Frontiers. Springer, Berlin, Heidelberg.

Lebon, G. 2014. Heat conduction at micro and nanoscales: a review through the prism of extended irreversible thermodynamics. J. Non-Equilib. Thermodyn. 39: 35-59.

Lhuillier, D. 1979. Stress tensor of dilute polymer solutions. Phys. Fluids 22: 2033-2035.

Liu, I.S. 1972. Method of Lagrange multipliers for exploitation of the entropy principle. Arch. Rat. Mech. Anal. 46: 131-148.

Liu. I.S. and I. Müller. 1983. Extended thermodynamics of classical and degenerate gases. Arch. Rat. Mech. Anal. 83: 285-332.

Liu, I.S., I. Müller and T. Ruggeri. 1986. Relativistic thermodynamics of gases. Ann. Phys. (New York) 169: 191-219.

Luikov, A.V. 1969. Analytical Heat Diffusion Theory. Acad. Press, New York.

Luzzi, R., A.R. Vasconcellos, J. Casas-Vázquez, and D. Jou. 1997. Characterization and measurement of a nonequilibrium temperature-like variable in irreversible thermodynamcs. Physica A 234: 699-714.

Luzzi R., A.R. Vasconcellos and J.S. Ramos. 2001. On the statistical foundations of irreversible thermodynamics. Teubner Verlag, Berlin.

Luzzi R., A.R. Vasconcellos and J.S. Ramos. 2002. Predictive statistical mechanics: a nonequilibrium ensemble formalism. Kluwer, Dordrecht.

Machlup, S. and L. Onsager. 1953. Fluctuations and irreversible process. II Systems with kinetic energy. Phys. Rev. 91: 1512-1515.

Mandel, J. 1978. Propriétés Mécaniques des Matériaux. Eyrolles, Paris.

Maugin, G. and W. Muschik. 1994. Thermodynamics with internal variables. J. Non- Equilib. Thermodyn. 19: 217-249 and 19: 250-289.

Maugin, G. 1999. The Thermodynamics of Nonlinear Irreversible Behaviours. World Scientific, Singapore. 
Maxwell, J.C. 1867. On the dynamical theory of gases. Philos. Trans. Roy. Soc. London, 157: $49-88$.

Meixner, J. and H.G. Reik. 1959. Thermodynamik der Irreversible Prozesse. In: Handbuch der Physik, Bd 3/ II. Springer, Berlin.

Meixner, J. 1968. TIP has many faces. In: Proceed. IUTAM Symposium Vienna, 1966. Springer, Berlin.

Meixner, J. 1974. Coldness and temperature, Arch. Rat. Mech. Anal. 3: 281-290.

Mongiovi, M.S. 1991. Superfluidity and the entropy conservation in extended thermodynamics. J. Non- Equilib. Thermodyn. 16: 225-239.

Mongiovi, M.S. 1992. Thermomechanical phenomena in extended thermodynamics of an ideal monatomic superfluid. J. Non-Equilib. Thermodyn. 17: 183-186.

Müller, I. 1966. Zur Ausbreitungsgeschwindigkeit von Störungen in kontinuierlichen Medien. Ph.D. Thesis, Technical University, Aachen.

Müller, I. 1967. Zum Paradox der Wärmetheorie. Z. Phys. 198: 329-344.

Müller, I. 1969. Toward relativistic thermodynamics. Arch. Rat. Mech. Anal. 34: 259-282.

Müller, I. 1970. Die Kälte funktion, eine univeselle Funktion in der Thermodynamik in der viskoser warmeleitender Flüssigkeiten. Arch. Rat. Mech. Anal. 40: 1-36.

Müller, I. 1971. The coldness, a universal function in thermoelastic bodies. Arch. Rat. Mech. Anal. 41: 319-332.

Müller, I. and T. Ruggeri. 1993. Extended Thermodynamics. Springer, New York.

Müller, I. and Ruggeri, T. 1998. Rational Extended thermodynamics. Springer, New York.

Müller, I., D. Reitebuch and W. Weiss. 2003. Extended thermodynamics consistent in order of magnitude. Continuum Mech. Thermodyn. 15: 113-146.

Müller, I. and W. Weiss. 2012. Thermodynamics of irreversible processes-past and present. Eur. Phys. J. H 37: 139-236.

Muschik, W. 1977. Empirical foundation and axiomatic treatment of non-equilibrium temperature. Arch. Rat. Mech. Anal. 66: 379-400.

Muschik, W. 2007. Why so many "Schools" of thermodynamics? Forsch. Ingenieurwes. 71: 149-161.

Nettleton, R.E. 1959. Thermodynamics of viscoelasticity in liquids. Phys. Fluids 2: 256-263.

Nettleton, R.E. 1960. Relaxation theory of thermal conduction in liquids. Phys. Fluids 3: $216-225$.

Nettleton, R.E. 1984. Early applications of extended irreversible thermodynamics. In: Lecture Notes in Physics, Vol. 199, pp. 1-31. Springer, Berlin.

Nettleton, R.E. and S.I Sobolev. 1995. Applications of extended thermodynamics to chemical, rheological, and transport process; a special survey Parts I and II. J. Non-Equilib. Thermodyn. 20: 205-229 and 20: 297-331.

Newton, I. 1701. Scala graduum caloris. Calorum descriptions \&signa. Phil. Trans. Royal Society London 22: 824-829. English translation in: Newton, I. 1809. Phil. Trans. Royal Society London 4: 572-575.

Newton, I. 1726. Philosophiae Naturalis Principia Mathematica, 3rd edition. Londini Juffia Societatis Regis ac Typis.

Nicolis, G. and I. Prigogine. 1977. Self-organization in Nonequilibrium Systems. Wiley, New York.

Nicolis, G. and I. Prigogine. 1989. Exploring Complexity. Freeman, New York.

Noll, W. and C. Truesdell. 1965. The non-linear field theories of mechanics. Springer, New York.

Ohm, G.S. 1827. Die galvanische Kette, mathematisch bearbeitet. T.H. Riemann, Berlin.

Onsager, L. 1931. Reciprocal relations in irreversible processes. Phys. Rev. 37: 405-426 and 38: 2265-2279.

Öttinger, H.C. 2005. Beyond Equilibrium Thermodynamics. Wiley, Hoboken.

Pavon, D., D. Jou and J. Casas-Vazquez. 1980. About the relativistic temperature gradient. Phys. Lett. A 78: 317-318.

Peltier, J.C. 1839. Observations sur les multiplicateurs et sur les piles thermo-électriques. Imprimerie E.J. Bailly, Paris.

Prigogine, I. 1947. Etude Thermodynamique des Phénomènes Irréversibles. Desoer, Liège. 
Prigogine, I. 1961. Introduction to Thermodynamics of Irreversible Processes. Interscience, New York.

Prigogine, I. 1980. From Time to Becoming. Time and Complexity in the Physical Sciences. Freeman, San Francisco.

Reichl, L.E. 1980. A Modern Course in Statistical Physics. Univ. Texas Press, Austin, Texas.

Rubi, M. and J. Casas-Vazquez. 1980. Thermodynamical aspects of micropolar fluids. J. Non-Equilib. Thermodyn. 5: 155-164.

Ruggeri, T. 1993. Recent results on wave propagation in continuous media. In: CISM Courses and Lectures, Vol. 344, pp. 105-154. Springer, Wien, New York.

Seebeck, T.J. 1821. Über den Magnetism der Galvanische Kette. K. Akad. Wiss. Berlin.

Sieniutycz, S. 1984. Variational approach to extended irreversible thermodynamics of heat and mass transfer. J. Non-Equilib. Thermodyn. 9: 61-71.

Sieniutycz, S. and P. Salomon. (eds.). 1992. Extended thermodynamics systems. Taylor and Francis, New York.

Sieniutycz, S. 1994. Conservation Laws in Variational Thermo-Hydrodynamics. Springer, Berlin.

Soret, C. 1879. Sur l'état d'équilibre que prend au niveau de sa concentration une dissolution saline primitivement homogène dont deux parties sont portées à des températures différentes. Arch. Sci. Phys. Nat. Genève 2: 187.

Stewart, J.M. 1977. Non-transient relativistic thermodynamics and kinetic theory. Proc. Roy. Soc. London A 357: 59-75.

Stocker, H. and W. Greiner. 1986. High energy heavy ion collisions-probing the equation of state of highly excited hadronic matter. Phys. Rep. 137: 277-392.

Stokes, G.C. 1851. On the effect of the internal friction of fluids on the motion of the pendulums. Transactions of the Cambridge Phil. Soc. IX: 8-106.

Struchtrup, H. 2005. Macroscopic Transport Equations for Rarefied Gas Flows. Springer, Berlin, New York.

Thomson, W. 1848. On an absolute thermometric scale founded on Carnot's theory of the motive power of heat and calculated from Regnault's observations. Phil. Mag. 33: 313317.

Thomson, W. 1851. On the dynamical theory of heat, with numerical results deduced from Mr Joule's equivalent of a thermal unit, and M. Regnault's observations on steam. Transactions of the Royal Society of Edinburgh XX (part II): 261-268 and 289-298.

Thomson, W. 1854. Account of experimental investigations to answer questions originating in the mechanical theory of thermoelectric currents. Edinburgh Roy. Soc. Proc. III: 255.

Truesdell, C. 1966. Six Lectures on Modern Natural Philosophy. Springer, Heidelberg.

Truesdell, C. 1969. Rational Thermodynamics. MacGraw Hill, New York.

Truesdell, C. and W. Noll. 1965. The Non-Linear Field Theories. In: Handbuch der Physik, Bd. III/3. Springer, Berlin.

Turing, A. 1952. The chemical basis of morphogenesis. Philos. Trans. R. Soc. London B 237: $37-72$.

van Kampen, N.G. 1987. Chapman-Enskog as an application of the method for eliminating fast variables. J. Stat. Phys. 46: 709-727.

Velasco, R.M. and L.S. Garcia-Colin. 1993. The kinetic foundations of non-local nonequilibrium thermodynamics. J. Non-Equilib. Thermodyn. 18: 157-172.

Verhas, J. 1983. On the entropy current. J. Non-Equilib. Thermodyn. 8: 201-206.

Vernotte, P. 1958. La véritable équation de la chaleur. Compt. Rend. Acad. Sci. Paris 247: 2103-2107.

Vidal, C., G. Dewel and P. Borkmans. 1994. Au-delà de l'Equilibre. Hermann, Paris.

Walgraef, D. 1997. Spatio-Temporal Pattern Formation. Springer, New York.

Wei, J. 1966. Irreversible thermodynamics in Engineering. Ind. Eng. Chem. 58: 55-60.

Weiss, W. 1990. Hierarchie der Erweiterten Thermodynamik. Dissertation Tech. Univ. Berlin.

Wilhelm, H.E. and S.H. Choi. 1975. Nonlinear hyperbolic theory of thermal waves in metals. J. Chem. Phys. 63: 2119-2123.

Woods, L.C. 1981. The bogus axioms of continuum mechanics. Bull. Inst. Math. Appl. 17: 98-102 and 1982. 18: 64-67. 